\title{
A Spin-Glass Model with Random Couplings
}

\author{
P. Collet ${ }^{1}$ and J.-P. Eckmann ${ }^{2, \star}$ \\ 1 Ecole Polytechnique, F-91120 Palaiseau, France \\ 2 IHES, F-91440 Bures-sur-Yvette, France
}

\begin{abstract}
We define a frustrated spin-glass model for which the MigdalKadanoff renormalization group is exact. Our model has random couplings, and the renormalization group acts on these. We study the high and low temperature phases of the model, exhibit a critical fixed point (in high dimension), and show that the Edwards-Anderson parameter takes a non-zero value in the low-temperature phase.
\end{abstract}

\section{Introduction}

In this paper, we analyze a model of spin-glasses which is similar to one studied before in a joint paper with Glaser and Martin [CEGM]. While that paper was concerned with what might be called the low-dimensional case, the present paper deals with the high-dimensional limit. In order to make this paper readable independently of [CEGM], we have chosen to repeat more or less verbally some of the introductory parts of that paper. We want to view the spin-glass problem as a problem of random variables describing random couplings. In particular, we are interested in the behavior of the effective random coupling under a change of scale. This will lead us naturally to a renormalization group (RG) approach.

This description will become exact in the hierarchical approximation described below, see also [GK], and we shall describe and study some aspects of the corresponding models which are random versions of a Migdal-Kadanoff type recursion relation $[\mathrm{K}, \mathrm{M}]$. Alternately, our approach leads to a study of nonindependent (but not strongly coupled (mean-field) [SK]) random variables, and our results can be viewed as an example of non-trivial behavior in this field of mathematics.

The purpose of our paper is to describe and analyze a class of such models, and, in particular, to study the "evolution" of the effective random coupling as a function of the size of the lattice (Sects. 2-5).

* On leave from the University of Geneva 

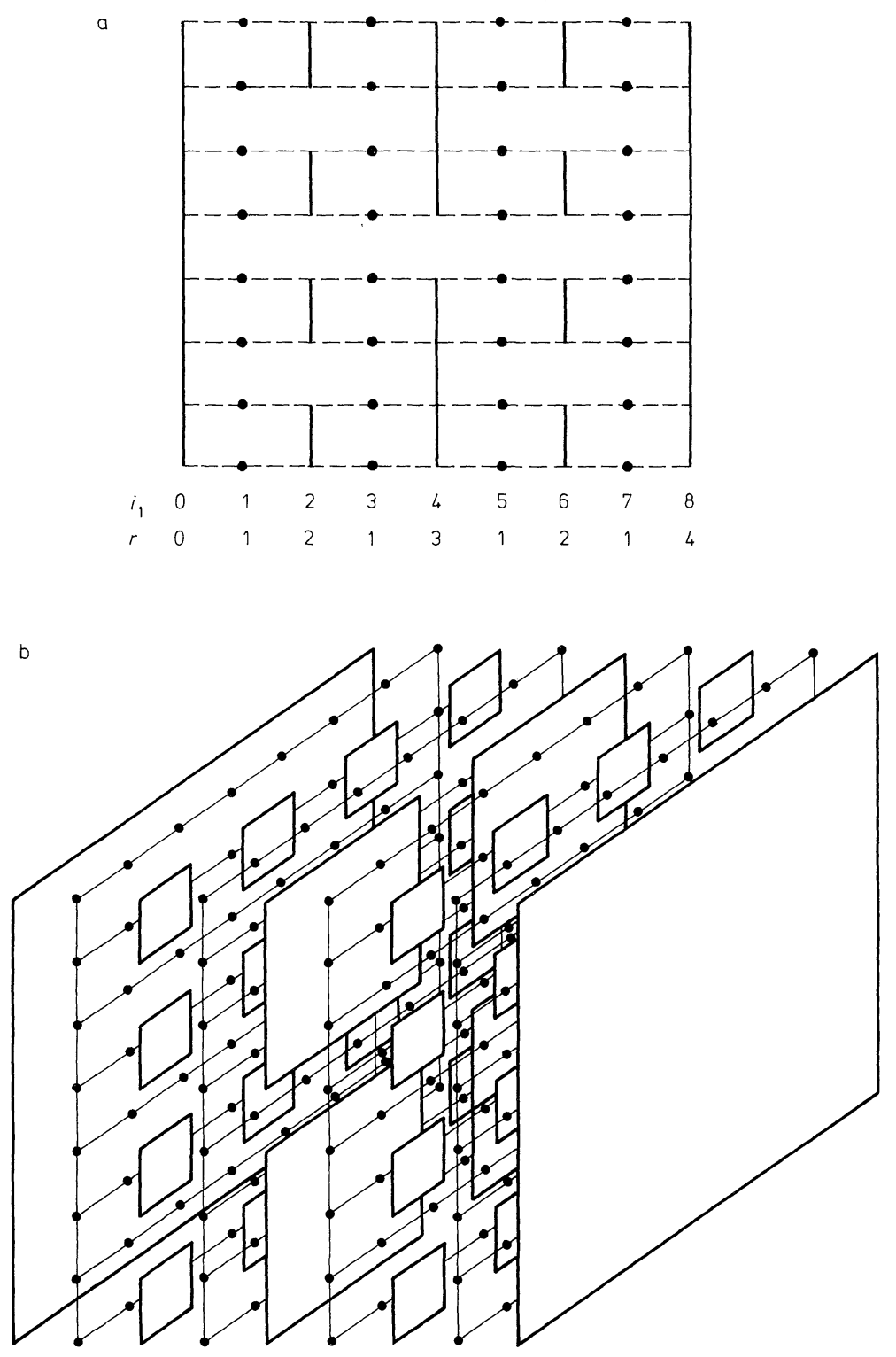

Fig. 1. a Lattice for $d=2, N=3$, e horizontal. b Lattice for $d=3, N=3$, e horizontal

In Sect. 7, we shall then arrive at the proof of existence of a spin-glass transition in the following sense. For a class of random interactions, we show that at high temperature, the expected value of the spin is $E(\langle s\rangle)=0$ and $E\left(\langle s\rangle^{2}\right)=0$, where \langle\rangle denotes the partition sum and $E(\cdot)$ denotes averaging the sample space of random couplings. At low temperature, we have $E(\langle s\rangle)=0$, but $E\left(\langle s\rangle^{2}\right) \neq 0$, and $E\left(\langle s\rangle^{2}\right) \rightarrow 1$ as $\beta \neq 0$, indicating a transition of the Edwards-Anderson parameter. 
In Sect. 6, we prove the existence of a critical fixed point for the renormalization group transformation. There is one relevant direction with eigenvalue $\sim 2$, and no marginal directions. Using general results [CEL] it would also be possible to show the existence of a critical surface.

\section{The Models}

The models we are considering have two equivalent formulations: One is based on a construction of Migdal and Kadanoff $[\mathrm{M}, \mathrm{K}]$, while the other is based on the recursive diamond shaped lattices (see $[\mathrm{GK}]$ ).

In the first case, we consider a $\mathbb{Z}^{d}$-lattice with an Ising spin at each site. In this lattice, we single out a direction, for example the first coordinate $\mathbf{e}=(1,0, \ldots, 0)$ and we assume that the system has side $2^{N}$ in the e-direction and $2^{N}-1$ in the others. The following description should be easier to understand by referring to Fig. 1.

To each (horizontal) link of the form $\mathbf{i}, \mathbf{i}+\mathbf{e}, \mathbf{i} \in \mathbb{Z}^{d}$ there is a random coupling $\xi_{\mathbf{i}}$. Below, we shall specify the nature, independence, ... of the random variables $\xi_{\mathbf{i}}$. There are now two types of interaction between the Ising spins.

i) The interaction energy between $s_{\mathbf{i}}$ and $s_{\mathbf{i}+\mathbf{e}}$ is $\xi_{\mathbf{i}}$.

ii) Every hyper"plane" with fixed first coordinate $i_{1}>0$ is partioned into $2^{(N+1-r)(d-1)}$ hypercubes of dimension $d-1$ and of side $2^{r}-1$, where $r$ is given by

$$
i_{1}=2^{r}+2^{r_{2}}+\ldots+2^{r_{k}}, \quad r<r_{2}<r_{3}<\ldots<r_{k}
$$

$i_{1}=0$ is handled as $i_{1}=2^{N}$. There is an infinite ferromagnetic coupling in each hypercube, i.e. all spins in one such hypercube are equal.

For a fixed choice of the random variables $\xi=\left\{\xi_{\mathbf{i}}\right\}$, we denote by $H_{N}(\mathbf{s}, \xi)$ the energy of the spin configuration $\mathbf{s}$. The Gibbs density at inverse temperature $\beta$ is given by

$$
G_{N}(\mathbf{s}, \xi, \beta)=\frac{e^{-\beta H_{N}(\mathbf{s}, \boldsymbol{\xi})}}{\sum_{\mathbf{s}^{\prime}} e^{-\beta H_{N}\left(\mathbf{s}^{\prime}, \boldsymbol{\xi}\right)}} .
$$

We are interested in the properties of $G_{N}$ as a function of $\beta$ in the thermodynamic limit, $N \rightarrow \infty$.

We now give a second description of the model, using a recursive buildup of a (hierarchical) lattice. This formulation has a natural extension to non-integer dimensions, as we shall see. One first chooses an integer $n \geqq 1$ (one should think of $n=2^{d-1}$, in the first formulation of the model). The lattice is then formed recursively as follows. The first lattice is formed by two sites and one link.

Fig. 2. One bond

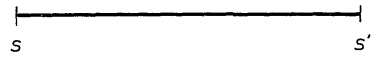

We call this $L_{0}$. If $L_{p}, p \geqq 0$ has been constructed, then $L_{p+1}$ is obtained by replacing each link by $n$ sites and $2 n$ links connecting each new site to the two ends of original link (see Fig. 3).

We now consider $L_{N}$. To each site $i$ (numbered in some suitable fashion) we associate an Ising spin, and to each link $(i, j)$ a random coupling constant $\xi_{i, j}$. 
Fig. 3. Increasing the level by one

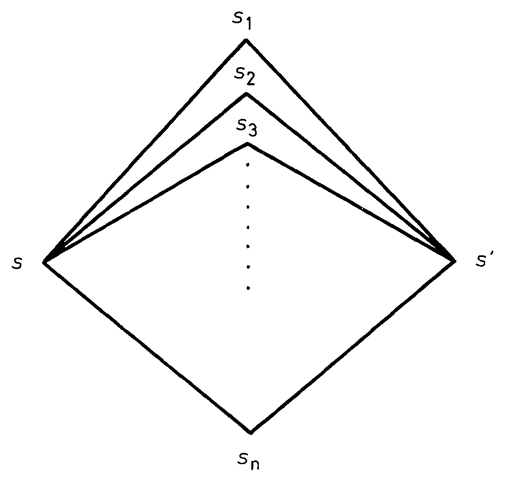

Again, for each fixed choice of the random variables, we can define

$$
H_{N}(\mathbf{s}, \xi)=\sum_{\substack{i, j \\ \text { (nearest neighbors) }}} \xi_{i, j} s_{i} s_{j} .
$$

The Gibbs measure is defined as before.

So far, we have said nothing about the nature of the random variables, and the model still leaves us some freedom of choice. In this paper, we choose each $\xi_{i, j}$ as an independent copy of a fixed random variable $\eta_{0}$. We assume that $\eta_{0}$ has finite variance, and, without loss of generality, that $\eta_{0}$ has variance 1 (this can be obtained by a change of the temperature scale). We shall also assume that the random variables have even distributions, and (for most of the results) that they have bounded densities. (This excludes Ising-like interactions.)

The above models are parametrized by $n$ and $\eta_{0}$. They possess frustration, and, by construction, the Migdal renormalization transformation [M] is exact. We also would like to refer to [BB] for numerical studies for such models.

\section{Renormalization Transformation}

In this section, we establish Migdal's recursion relations, which are exact for the models we have described. Consider $L_{N}$. The renormalization consists in integrating over all spins introduced in the step leading from $L_{N-1}$ to $L_{N}$. The

Fig. 4. Labelling one diamond

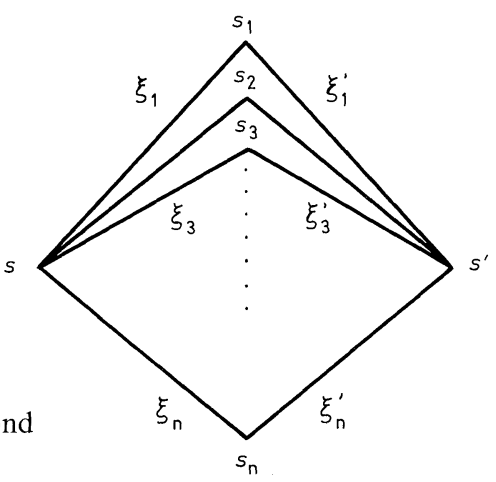


resulting lattice $L_{N-1}$ will have new effective (random) coupling constants. We now derive the formula for getting the new couplings $\hat{\xi}$ as a function of the old ones. It is clearly sufficient to consider the following situation.

We reemphasize that we want to sum over $s_{1}, \ldots, s_{n}$ and replace $\xi_{1}, \ldots, \xi_{n}$, $\xi_{1}^{\prime}, \ldots, \xi_{n}^{\prime}$ by a new random variable $\hat{\xi}$ giving an effective coupling between $s$ and $s^{\prime}$.

It is useful to introduce the random variables $x_{i}=\tanh \left(\xi_{i}\right), x_{i}^{\prime}=\tanh \left(\xi_{i}^{\prime}\right)$. Moreover, we shall assume that the inverse temperature $\beta$ has been absorbed in the definition of the $\xi_{i}$. We then have to compute

$$
\begin{aligned}
I & =\sum_{\substack{s_{1}, \ldots \ldots, s_{n} \\
= \pm 1}} \prod_{i=1}^{n}\left(e^{\xi_{i} s_{i}} e^{\xi_{i} s_{i} s^{\prime}}\right) \\
& =\sum_{\substack{s_{1}, \ldots, s_{n} \\
= \pm 1}} \prod_{i=1}^{n} \cosh \xi_{i} \cdot \cosh \xi_{i}^{\prime} \cdot\left(1+x_{i} s s_{i}\right)\left(1+x_{i}^{\prime} s_{i} s^{\prime}\right) .
\end{aligned}
$$

The factors $\cosh \xi_{i} \cdot \cosh \xi_{i}^{\prime}$ will eventually disappear in the normalization of the partition function. We shall omit them, henceforth. The quantity to study is thus

$$
\begin{aligned}
\prod_{i=1}^{n} & \sum_{s_{i}= \pm 1}\left(1+x_{i} s s_{i}\right)\left(1+x_{i}^{\prime} s_{i} s^{\prime}\right) \\
= & 2^{n} \prod_{i=1}^{n} \frac{1}{\cosh \left(\tanh ^{-1}\left(x_{i} x_{i}^{\prime}\right)\right)} \\
& \cdot \prod_{i=1}^{n}\left(\cosh \left(\tanh ^{-1}\left(x_{i} x_{i}^{\prime}\right)\right)+\sinh \left(\tanh ^{-1}\left(x_{i} x_{i}^{\prime}\right)\right) s s^{\prime}\right) \\
= & 2^{n} \prod_{i=1}^{n} \frac{1}{\cosh \left(\tanh ^{-1}\left(x_{i} x_{i}^{\prime}\right)\right)} \exp \left(\sum_{i=1}^{n} \tanh ^{-1}\left(x_{i} x_{i}^{\prime}\right) s s^{\prime}\right) \\
= & 2^{n} \cosh \left(\sum_{i=1}^{n} \tanh ^{-1}\left(x_{i} x_{i}^{\prime}\right)\right) \frac{1+\tanh \left(\sum_{i=1}^{n} \tanh ^{-1}\left(x_{i} x_{i}^{\prime}\right)\right) s s^{\prime}}{\prod_{i=1}^{n} \cosh \left(\tanh ^{-1}\left(x_{i} x_{i}^{\prime}\right)\right)} .
\end{aligned}
$$

We again omit the factors which do not depend on $s, s^{\prime}$ (and which disappear in the normalization), and we get the transformation for the $\xi$ :

$$
\xi=\sum_{i=1}^{n} \tanh ^{-1}\left(\tanh \left(\xi_{i}\right) \tanh \left(\xi_{i}\right)\right) .
$$

Note that $n$ should be thought of as $2^{d-1}$, where $d$ is the dimension of the lattice.

For completeness, we also give the renormalization of a correlation function, for one spin. The quantity is given by

$$
\begin{aligned}
J\left(s_{1}\right) & =\frac{1}{I} \sum_{s_{1}, \ldots, s_{n}} s_{1} \prod_{i=1}^{n} \cosh \left(\xi_{i}\right) \cosh \left(\xi_{i}^{\prime}\right)\left(1+x_{i} s s_{i}\right)\left(1+x_{i}^{\prime} s_{i} s^{\prime}\right) \\
& =\frac{x_{1}\left(1-x_{1}^{\prime 2}\right) s+x_{1}^{\prime}\left(1-x_{1}^{2}\right) s^{\prime}}{1-x_{1}^{2} x_{1}^{\prime 2}} \cdot\left(1+\tanh \left(\sum_{i=1}^{n} \tanh ^{-1}\left(x_{i} x_{i}^{\prime}\right)\right) s s^{\prime}\right) .
\end{aligned}
$$


The identities (3.1) and (3.2) will allow us to compute explicitly the models $L_{N}$ by $N$-fold iteration, and we shall be able to take the thermodynamic limit, $N \rightarrow \infty$.

Note. We will, henceforth, write th instead of tanh.

Having described the general features of the model, we now describe in more detail its temperature dependence and the renormalization transformation, that is, the effect of summing over the spins at a given level. We also introduce at this stage some notation.

We assume that the random coupling has a probability law of a random variable $\eta_{0}$, with variance 1 . The coefficient in front of the spins at inverse temperature $\beta$ is then $\xi_{0}=\beta \eta_{0}$. Given a random coupling $\xi$, the renormalization group induces a new random coupling - at the next level - which we call $\hat{\xi}$, in two steps, as can be easily derived from (3.1). Namely, we construct $\tilde{\xi}=\operatorname{th}^{-1}\left(\operatorname{th}(\xi) \operatorname{th}\left(\xi^{\prime}\right)\right)$, where $\xi^{\prime}$ is an independent copy of $\xi=\xi_{0}$ and

$$
\xi=\sum_{i=1}^{n} \xi^{(i)},
$$

where the $\tilde{\xi}^{(i)}, i=1, \ldots, n$ are independent copies of $\tilde{\xi}$. The number $n$ is a constant of each individual model $\left(\sim 2^{\text {dimension }-1}\right)$. We regard it as fixed, and sufficiently large throughout, without stating this condition every time. Note that the conditions on the largeness of $n$ will depend on $\eta_{0}$. We also denote $\mathscr{N} \xi=\xi$, i.e. $\mathscr{N}$ is a map from random variables to random variables (depending on $n$ ).

We shall adopt the following conventions: $f, \tilde{f}, \hat{f}$ denote, respectively the densities of $\xi, \tilde{\xi}, \hat{\xi}$ and similarly, for the variances $\sigma, \tilde{\sigma}, \hat{\sigma}$.

We next describe heuristically the behavior of the random variables under renormalization. Assume $\xi$ has small variance, $\sigma$, and assume $n$ is large. Since the variance is small, $\xi$ is small, and hence, $\widetilde{\xi}=\operatorname{th}^{-1}\left(\operatorname{th}(\xi) \operatorname{th}\left(\xi^{\prime}\right)\right) \sim \xi^{\prime}$. Therefore, $\tilde{\sigma}^{2}=E\left(\tilde{\xi}^{2}\right) \sim E\left(\xi^{2} \xi^{\prime 2}\right)=\sigma^{4}$. Since $\hat{\xi}$ is the sum of $n$ independent copies of $\tilde{\xi}$, we have the renormalization group relation for the variance:

$$
\hat{\sigma} \sim n^{1 / 2} \sigma^{2} .
$$

The fixed point of this relation is $\sigma=n^{-1 / 2}$ (thus, $\sigma$ is small consistently if $n$ is large), and the unstable eigenvalue at the fixed point is

$$
\left.\frac{d \hat{\sigma}}{d \sigma}\right|_{\sigma=n^{-1 / 2}} \sim 2 \sigma n^{1 / 2}=2 .
$$

This paper makes these heuristic observations precise.

\section{Low-Temperature Behavior}

We want to show that given $\eta_{0}$, the random coupling, of variance 1 , there is a $\beta_{0}$ such that for every $\beta>\beta_{0}$, one has

$$
\operatorname{th}\left(\left|\mathscr{N}^{p}\left(\beta \eta_{0}\right)\right|\right) \rightarrow 1, \text { almost surely as } \quad p \rightarrow \infty .
$$

Define $Q=E\left(\inf \left(\eta_{0}^{2}, \eta_{0}^{\prime 2}\right)\right) / 8$, where $E$ means expectation and $\eta_{0}^{\prime}$ is an independent copy of $\eta_{0}$. Note that $Q \neq 0$, since $\eta_{0}$ has non-zero variance. 
Theorem 4.1. There is a constant $N_{0}=N_{0}(Q)>320^{2}$ such that for sufficiently large $\beta$ and for $n>N_{0}$ the sequences $\xi_{p}(\beta):=\mathscr{N}^{p}\left(\beta \eta_{0}\right)$ satisfy

1) $\sigma_{p}:=\operatorname{var}\left(\xi_{p}(\beta)\right)>\left(n^{1 / 2} / 320\right)^{p} \beta Q^{1 / 2}$ (i.e. $\sigma_{p}$ diverges),

2) if the density of $\eta_{0}$ is in $L_{\infty}$ and $n$ is even, then the density $f_{p}$ of $\xi_{p}(\beta)$ satisfies for some universal constant $K$,

$$
\left|f_{p}(x)-\frac{1}{\left(2 \pi \sigma_{p}^{2}\right)^{1 / 2}} e^{-x^{2} / 2 \sigma_{p}^{2}}\right| \leqq \frac{K \sigma_{p}^{2}}{n^{1 / 2}\left(\sigma_{p}+|x|\right)^{3}} .
$$

Remarks. 1) With a little bit more work the condition " $n$ even" can be dropped.

2) The divergence of the variance as shown in Theorem 4.1.1 does not imply that the random variable is large (almost surely), since it could take with small probability a very large value, while with large probability it could take the value zero. The purpose of Theorem 4.1.2 is to exclude this possibility.

Proof. Denote by $\tilde{\xi}(\beta)$ the random variable, $\tilde{\xi}(\beta)=\operatorname{th}^{-1}\left(\operatorname{th}\left(\beta \eta_{0}\right)\right.$ th $\left.\left(\beta \eta_{0}^{\prime}\right)\right)$, where $\eta_{0}^{\prime}$ is an independent copy of $\eta_{0}$. We claim: For sufficiently large $\beta$, the variance $\tilde{\sigma}(\beta)$ of $\widetilde{\xi}(\beta)$ satisfies

$$
\tilde{\sigma}(\beta)^{2} \geqq \beta^{2} Q
$$

and

$$
\tilde{\alpha}(\beta):=E\left(|\widetilde{\xi}(\beta)|^{3}\right) / \tilde{\sigma}(\beta)^{3} \leqq 1 / Q^{3 / 2} .
$$

Indeed, by Lemma A1.2), we have

$$
\begin{aligned}
\tilde{\sigma}(\beta)^{2} & \geqq E\left(\left(\min \left(\left|\beta \eta_{0}\right|,\left|\beta \eta_{0}^{\prime}\right|\right)-\log 2\right)^{2} \chi_{\left|\beta \eta_{0}\right|>2 \log 2,\left|\beta \eta_{0}^{\prime}\right|>2 \log 2}\right) \\
& \geqq \frac{\beta^{2}}{4} E\left(\chi_{\left|\beta \eta_{0}\right|>2 \log 2,\left|\beta \eta_{0}^{\prime}\right|>2 \log 2} \min \left(\eta_{0}^{2}, \eta_{0}^{\prime 2}\right)\right) \\
& \geqq \beta^{2} Q,
\end{aligned}
$$

for sufficiently large $\beta$. Hence, (4.1) holds. To show (4.2), we apply Lemma A3, yielding

$$
\tilde{\alpha}(\beta) \leqq \beta^{3} E\left(\eta_{0}^{2}\right)^{3 / 2} / \tilde{\sigma}(\beta)^{3},
$$

by Hölder's inequality, and hence, (4.2) follows from (4.1). Thus, we conclude that for large $\beta$, the variance $\tilde{\sigma}(\beta)$ is large while the "skewness" $\tilde{\alpha}(\beta)$ is bounded.

We shall now use in alternation the following two lemmas. Lemma 4.2. Given a random variable $\tilde{\xi}$, and forming $\hat{\xi}=\sum_{i=1}^{n} \widetilde{\xi}^{(i)}$, the distribution
function $\hat{F}$ of $\xi$ satisfies

$$
\left|\hat{F}\left(x n^{1 / 2} \tilde{\sigma}\right)-\Phi(x)\right| \leqq \tilde{\alpha} / n^{1 / 2},
$$

where $\Phi$ is the normal distribution.

This is a reformulation of the Berry-Esseen inequality (see e.g. [P, pp. 111, 128, No. 8]).

Note that

$$
n^{1 / 2} \tilde{\sigma}=\operatorname{var}(\hat{\xi})
$$


In the next lemma, we control the variance of $\tilde{\xi}$ relative to that of $\xi$, measuring the "skewness" caused by $\operatorname{th}^{-1}(\operatorname{th}(\xi) \operatorname{th}(\xi))$.

Lemma 4.3. Assume that the distribution $F$ of $\xi$ satisfies $|F(x \sigma)-\Phi(x)| \leqq 1 / 40$ and $\sigma>9$. Then $\tilde{\sigma} \geqq \sigma / 320$ and $\tilde{\alpha} \leqq 320^{3}$.

Proof. We use the normalized variables $\eta=\xi / \sigma, \eta^{\prime}=\xi^{\prime} / \sigma$. We bound $\tilde{\sigma}^{2}$ by

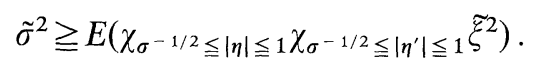

We recall the inequality Lemma A1.2). It implies

$$
\begin{aligned}
\tilde{\sigma}^{2} a g 1 & \geqq \int_{\sigma^{-1 / 2} \leqq y, y y^{\prime} \leqq 1}\left(\inf \left(\sigma y, \sigma y^{\prime}\right)-\log 2\right)^{2} d F(y \sigma) d F\left(y^{\prime} \sigma\right) \\
& \geqq \int_{1 / 3 \leqq y, y^{\prime} \leqq 1}\left(\inf \left(\sigma y, \sigma y^{\prime}\right)-\log 2\right)^{2} d F(y \sigma) d F\left(y^{\prime} \sigma\right) \\
& \geqq \sigma^{2}(1 / 3-(\log 2) / \sigma)^{2}\left[F(\sigma)-F\left(\frac{\sigma}{3}\right)\right]^{2} .
\end{aligned}
$$

By assumption,

$$
\begin{aligned}
F(\sigma)-F\left(\frac{\sigma}{3}\right) & \geqq \Phi(1)-\Phi\left(\frac{1}{3}\right)-1 / 20 \\
& \geqq 0.2-1 / 20 \\
& \geqq 1 / 4 .
\end{aligned}
$$

The first assertion follows. By the proof of $(4.3), E\left(|\tilde{\xi}|^{3}\right) \leqq \sigma^{3}$. Since $\tilde{\alpha}=E\left(|\tilde{\xi}|^{3}\right) / \tilde{\sigma}^{3}$, the second assertion follows from the first.

Proof of Theorem 4.1.1. Choose $N_{0}=\max \left(1600 / Q^{3},\left(40 \cdot 320^{3}\right)^{2}\right)$. By (4.1), (4.2) we find for $n>N_{0}: \tilde{\sigma}^{2} \geqq \beta^{2} Q, \tilde{\alpha} \leqq 1 / Q^{3 / 2}$. By Lemma 4.2 and Eq. (4.4), we have for $\xi_{1}:=\xi(\beta)=\mathcal{N}\left(\beta \eta_{0}\right): \sigma_{1}=\operatorname{var}\left(\xi_{1}\right)=n^{1 / 2} \tilde{\sigma}>9$ for large $\beta$, and for its distribution function $F_{1},\left|F_{1}\left(x \sigma_{1}\right)-\Phi(x)\right|<1 / 40$. We apply Lemma 4.3 to $\xi_{1}$ and obtain for $\tilde{\xi}_{1}$ : $\tilde{\sigma}_{1}=\operatorname{var}\left(\widetilde{\xi}_{1}\right)>\sigma_{1} / 320$ and $\tilde{\alpha}_{1} \leqq 320^{3}$. We reapply Lemma 4.2 and we find for $\xi_{2}=\mathscr{N}\left(\xi_{1}\right)=\mathscr{N}^{2}\left(\beta \eta_{0}\right)$ :

$$
\sigma_{2}=n^{1 / 2} \tilde{\sigma}_{1} \geqq n^{1 / 2} \sigma_{1} / 320
$$

and

$$
\left|F_{2}\left(x \sigma_{2}\right)-\Phi(x)\right| \leqq 320^{3} / n^{1 / 2}<1 / 40 .
$$

We have completed an induction step and 1) follows.

Proof of Theorem 4.1.2. If the density $g_{0}$ of $\eta_{0}$ is in $L_{\infty}$, then the density $g_{0}(\beta)$ of $\beta \eta_{0}$ is $\beta^{-1} g_{0}(x / \beta)$, and hence, $\left\|g_{0}(\beta)\right\|_{\infty}=\beta^{-1}\left\|g_{0}\right\|_{\infty}$. We apply Lemma A2 with $f=g=g_{0}$, noting that $\|f\|_{\infty}<2$ if $\beta$ is large. Hence, the density $\tilde{f}$ of $\widetilde{\xi}(\beta)$ satisfies

$$
\|\tilde{f}\|_{1}=1, \quad\|\tilde{f}\|_{2} \leqq C\left\|g_{0}\right\|_{\infty}^{1 / 2} / \beta^{1 / 2} .
$$

From this, we conclude that

$$
\|\tilde{f} * \tilde{f}\|_{1} \leqq 1 \text { and }\|\tilde{f} * \tilde{f}\|_{\infty} \leqq C^{2}\left\|g_{0}\right\|_{\infty} / \beta .
$$


Note that $\hat{f}=(\tilde{f} * \widetilde{f})^{* n / 2}$, since $\tilde{f} * \tilde{f}$ is nothing else than the density of $\tilde{\xi}+\widetilde{\xi}^{\prime}$, with $\tilde{\xi}^{\prime}$ an independent copy of $\widetilde{\xi}$. It is easy to see that $E\left(\left|\tilde{\xi}+\widetilde{\xi}^{\prime}\right|^{3}\right) \leqq 8 E\left(|\widetilde{\xi}|^{3}\right)$ and $E\left(\left(\widetilde{\xi}+\widetilde{\xi}^{\prime}\right)^{2}\right)$ $=2 \tilde{\sigma}^{2}(\beta)$. Therefore,

$$
\frac{E\left(\left|\tilde{\xi}+\tilde{\xi}^{\prime}\right|^{3}\right)}{E\left(\left(\tilde{\xi}+\tilde{\xi}^{\prime}\right)^{2}\right)^{3 / 2}} \leqq 4 \tilde{\alpha}(\beta)
$$

Thus, by our previous results, $\tilde{\xi}+\widetilde{\xi}^{\prime}$ is a random variable with variance $2 \tilde{\sigma}^{2}(\beta)$ and skewness less than $1 / Q^{3 / 2}$. We apply to this random variable Sahaidarova's result $[$ P, p. 214, No. 5], which states that for some universal $A$,

$$
\left|\hat{f}(x)-\left(2 \pi \hat{\sigma}^{2}\right)^{-1 / 2} e^{-x^{2} / 2 \hat{\sigma}^{2}}\right| \leqq \frac{A \tilde{\alpha}^{5} \max \left(1,\|\tilde{f} * \tilde{f}\|_{\infty}^{7}\right) \hat{\sigma}^{2}}{(n / 2)^{1 / 2}(\hat{\sigma}+|x|)^{3}} .
$$

Given our previous bounds, this implies the assertion of the theorem for $p=1$. Clearly, Eq. (4.5) implies, for large $\hat{\sigma}$, that $\|\hat{f}\|_{\infty}<2$, and hence, we can start a new induction step, applying Lemma A2 to $f=g=\hat{f}=: f_{1}$ and repeating the arguments given above. The proof is complete.

\section{High-Temperature Behavior}

We now want to show that given $\eta_{0}$ of variance 1 , at sufficiently high temperature the variance of $\mathscr{N}^{p}\left(\beta \eta_{0}\right)$ tends to zero as $p$ tends to infinity.

Theorem 5.1. Given $\eta_{0}$ of variance 1 and $n$, then for every $\beta<1 /(50 n)^{1 / 2}$, we have $\operatorname{var}\left(\mathcal{N}^{p}\left(\beta \eta_{0}\right)\right)^{2} \leqq 2^{-p} /(50 n)$.

Proof. We have $\operatorname{var}\left(\beta \eta_{0}\right)=\beta$. Set $\beta_{1}=1 /(50 n)^{1 / 2}$. If $\beta<\beta_{1}$, then $E\left(\left(\beta \eta_{0}\right)^{2}\right)=\beta^{2}$ $\leqq 1 /(50 n)$. Hence, Corollary A4 applies, and the assertion follows.

\section{Existence of a Critical Fixed Point}

In all of the following discussion, $n$ is supposed to be a fixed, large integer, and $\mathscr{N}$ will denote the operator $\mathscr{N}$ for that value of $n$.

If $\xi$ is a Gaussian random variable with (small) variance $\sigma$, then we expect $\hat{\xi}=\mathscr{N} \xi$ to be an "almost" Gaussian random variable with variance $\sim n^{1 / 2} \sigma^{2}$. Namely, in Proposition 6.6, we shall see that the variance $\tilde{\sigma}$ of $\tilde{\xi}$ is $\sim \sigma^{2}$, and the variance of $\hat{\xi}$ equals $n^{1 / 2} \tilde{\sigma}$. Thus, the fixed point of $\mathscr{N}$, if it exists, is an almost Gaussian random variable, with variance $\sim n^{-1 / 2}$. Furthermore, as we shall see below, the only unstable eigendirection of $D \mathscr{N}$ is, essentially, the variance, and it will be seen to have eigenvalue close to 2 . We choose our function space in a way to account for these facts.

Our basic space, $\mathscr{H}$, is decomposed as the square of the variance, and a component (infinite dimensional) describing the deviation from being Gaussian.

Definition.

$$
\begin{aligned}
\mathscr{H}= & \left\{(s, \varphi)\left|s \in \mathbb{R}, \varphi \in L_{\infty}(\mathbb{R}), \frac{n^{-1 / 4}}{\log n}\right| s \mid+\|\varphi\|_{\infty}+\left\|x^{4} \varphi\right\|_{2}<\infty,\right. \\
& \left.\int \varphi(x) x^{2} d x=0,\right\} .
\end{aligned}
$$


Here, and in the sequel, \|\|$_{p}$ denotes the $L_{p}$ norm, and $\left\|x^{k} \varphi\right\|_{p}$ is a shorthand notation for $\left(\int\left|x^{k} \varphi(x)\right|^{p} d x\right)^{1 / p}$. We denote by ||$_{\mathscr{C}}$ the norm on $\mathscr{H}$, and we write $|\varphi|_{\mathscr{\ell ^ { \prime }}}=\|\varphi\|_{\infty}+\left\|x^{4} \varphi\right\|_{2}$.

To every pair $(s, \varphi) \in \mathscr{H}$ with $s>0$ we associate a function $f$ by the identity

$$
f(x)=(n / s)^{1 / 2}\left(h\left(x(n / s)^{1 / 2}\right)+\varphi\left(n^{1 / 2} x\right)\right),
$$

where we adopt the convention $h(z):=(2 \pi)^{-1 / 2} e^{-z^{2} / 2}$, throughout. Thus, if $s=1$ and $\varphi \equiv 0$, then $f$ is a Gaussian with variance $n^{-1 / 2}$.

We define $\mathscr{H}_{1}=\left\{f \mid\|f\|_{\infty} n^{-1 / 2}+n^{7 / 4}\left\|x^{4} f\right\|_{2}<\infty\right\}$, and we denote by ||$_{\mathscr{H}_{1}}$ the corresponding norm. The operator $\mathscr{S}_{1}: \mathscr{H} \rightarrow \mathscr{H}_{1}$ is defined by the relation $\mathscr{J}_{1}(s, \varphi)=f$, as defined in (6.1). Note that $f$ is not necessarily the density of a probability law, but, for every $(s, \varphi) \in \mathscr{H}, s>0$, one has $\int f(x) d x=1$ and $\int x^{2} f(x) d x=s$. Thus, we have obtained our parametrization of "almost" Gaussian functions.

We next define the map $\mathscr{S}$ by

$$
\begin{aligned}
& \mathscr{S}(f)(x)=\tilde{f}(x)=\left(1-\operatorname{th}^{2} x\right) \int_{|\operatorname{th} x| \leqq|y| \leqq 1} \frac{d y}{|y|} \frac{1}{1-\frac{\operatorname{th}^{2} x}{y^{2}}} \frac{1}{1-y^{2}} \\
& f\left(\operatorname{th}^{-1}(y)\right) f\left(\operatorname{th}^{-1}\left(\frac{\operatorname{th} x}{y}\right)\right) .
\end{aligned}
$$

We shall see that it is useful to consider $\mathscr{S}$ as a map $\mathscr{H}_{1} \rightarrow \mathscr{K}$, where

$$
\mathscr{K}=\left\{\tilde{f} \mid\|\tilde{f}\|_{1}+n^{-1 / 2}(\log n)^{-1}\|\tilde{f}\|_{2}+n^{7 / 2}\left\|x^{4} \tilde{f}\right\|_{1}\right\} .
$$

This operator $\mathscr{S}$ does not commute with the change of scale depending on $n$, but we shall show that $\tilde{f}$ still "scales" reasonably when $n$ is changed.

We next consider the operation $\mathscr{T}: \mathscr{K} \rightarrow \mathscr{H}_{1}$, where $f(x)=\mathscr{T} \tilde{f}(x)=\tilde{f}^{* n}(x)$ (here, $f^{* p}$ is the $p$-fold convolution product).

Finally, we define $\mathscr{T}_{1}: \mathscr{H}_{1} \rightarrow \mathscr{H}$ by

$$
\begin{gathered}
s=n \int x^{2} f(x) d x, \\
\varphi=\left(\frac{s}{n}\right)^{1 / 2} \circ\left(\frac{x}{n^{1 / 2}}\right)-h\left(\frac{x}{s^{1 / 2}}\right) .
\end{gathered}
$$

Thus, $\mathscr{M}=\mathscr{T}_{1} \mathscr{T} \mathscr{S} \mathscr{S}_{1}$ is (supposedly) a map from part of $\mathscr{H}$ to $\mathscr{H}$. We shall show that $\mathscr{M}$ has a fixed point $\left(s_{*}, \varphi_{*}\right)$. Note also that $\mathscr{T}_{1}=\mathscr{S}_{1}^{-1}$, and in particular, that $\mathscr{N}=\mathscr{T} \mathscr{S}$ and $f_{*}=\mathscr{I}_{1}\left(s_{*}, \varphi_{*}\right)$ is a fixed point for $\mathscr{N}$. Furthermore, the spectrum of $D \mathscr{N}$ equals the spectrum of $D \mathscr{M}$.

Thus, it is sufficient to study $\mathscr{M}$, and we intend to show it has a fixed point near $(1,0)$. More specifically, we consider the ball $\mathscr{B} \subset \mathscr{H}$ :

$$
\mathscr{B}=\left\{\left.(s, \varphi)||(s-1, \varphi)\right|_{\mathscr{H}} \leqq(\log n)^{10} / n\right\} .
$$

Then we have

Theorem 6.1. The map $\mathscr{M}$ maps $\mathscr{B}$ to $\mathscr{H}$ and has a unique fixed point in $\mathscr{B}$. The map $D \mathscr{M}$ has a simple eigenvalue at $2+O\left(n^{-1 / 2}\right)$ and the remainder of the spectrum strictly inside the unit disk. 
From the definition of $\mathscr{B}$ it is clear that the fixed point is close to Gaussian, when $n$ is large.

We shall prove Theorem 6.1 by using a variant of Newton's method. The input to this method is given by

Theorem 6.2. 1) $|\mathscr{M}(1,0)-(1,0)|_{\mathscr{H}}=\mathcal{O}(\log n / n)$.

2) $\mathscr{M}$ is defined as a map from $\mathscr{B}$ to $\mathscr{H}$ and $D \mathscr{M}_{(s, \varphi)}$ is a $2 \times 2$ "matrix" whose elements

$$
\left[\begin{array}{ll}
A & B \\
C & D
\end{array}\right]
$$

satisfy:

$$
\begin{gathered}
A=2+\mathcal{O}\left(n^{-1 / 2}\right), \\
|B(0, \psi)|_{\mathscr{H}} \leqq n^{-1 / 4}(\log n)^{-1} \cdot n^{1 / 4}|(0, \psi)|_{\mathscr{H}}, \\
|C(t, 0)|_{\mathscr{H}} \leqq(\log n)^{4} / n^{1 / 2} \cdot n^{1 / 4} \cdot \log n|(t, 0)|_{\mathscr{H}}, \\
|D(0, \psi)|_{\mathscr{H}} \leqq(\log n)^{5} / n^{1 / 4}|(0, \psi)|_{\mathscr{H}} .
\end{gathered}
$$

These bounds hold uniformly for $(s, \varphi)$ in $\mathscr{B}$.

Proof of Theorem 6.1. Theorem 6.2 implies that for all $(s, \varphi) \in \mathscr{B}$,

$$
D \mathscr{M}_{(s, \varphi)}-\mathbb{1}_{\mathscr{H}}=\left[\begin{array}{cc}
1 & 0 \\
0 & -\mathbb{1}_{\mathscr{H}^{\prime}}
\end{array}\right]+\mathscr{M}_{(s, \varphi)}^{\prime}=: \mathscr{M}^{*}+\mathscr{M}_{(s, \varphi)}^{\prime},
$$

where $\mathscr{M}^{\prime}$ has norm less than $(\log n)^{-2}$. Therefore, if we define $\Phi=\mathbb{1}_{\mathscr{H}}-\mathscr{M}^{*-1}$ $\cdot\left(\mathscr{M}-\mathbb{1}_{\mathscr{H}}\right)$, we find that $D \Phi_{(s, \varphi)}$ has norm less than $1 / 2$ for $(s, \varphi) \in \mathscr{B}$. Combining Theorem 6.2.1 and 2, we see that $\Phi$ is a contraction from $\mathscr{B}$ into itself, and hence, it has a fixed point. Thus, $\mathscr{M}$ and in turn $\mathscr{N}$ have a fixed point as asserted. It also follows from Theorem 6.2 that $\mathscr{N}$ has a simple eigenvalue $2+\mathcal{O}\left(n^{-1 / 2}\right)$, and the remainder of the spectrum strictly inside the unit disk.

Remark. The bounds on $C, D$ in Theorem 6.2 are immediate consequences of Propositions 6.6 and 6.10.2). We bound $A$ in Lemma 6.11 and $B$ in Lemma 6.12.

Remark. The fixed point found above is a positive function. We only sketch the argument. There is a neighborhood $U$ of the fixed point of radius $\mathcal{O}\left(n^{-1 / 2}\right)$, where the tangent map is uniformly hyperbolic (easy from Theorem 6.2). Moreover, in $U$, the unstable direction stays inside a cone of aperture $\mathcal{O}\left(n^{-1 / 4}\right)$ with axis the second Hermite function. Moreover, since the stable part of the spectrum is inside a ball of radius $\mathcal{O}\left(n^{-1 / 4}\right)$, we deduce that the stable manifold extends to a distance $\mathcal{O}\left(n^{-1 / 2}\right)$ of the fixed point, and the tangent directions stay outside a cone of axis as above and aperture $\pi / 4$. This implies that the one parameter family $s \rightarrow s^{1 / 2} h\left(s^{1 / 2}\right)$ crosses transversally the stable manifold $\left[\right.$ at a distance $\mathcal{O}\left(\frac{(\log n)^{10}}{n}\right)$ by Theorem 6.1]. Let $s_{0}$ be the square of the variance of the intersection. Since $\mathscr{N}$ transforms positive functions into positive functions and $\mathscr{N}^{p}\left(s_{0}^{1 / 2} h\left(s_{0}^{1 / 2}\right)\right)$ converges to the fixed point, we deduce that it is a positive function. 
The proof of Theorems 6.2.1) and 6.2.2) is based on a careful study of the operators $\mathscr{S}$ and $\mathscr{T}$. (The considerations concerning $\mathscr{S}_{1}$ and $\mathscr{T}_{1}$ are of a simpler nature and will be done in a more ad hoc fashion.) Furthermore, we shall need some control over the variance.

The Operator $\mathscr{S}$. This is the net effect of summing over one spin, and it induces a highly non-linear (and non-Gaussian) effect on the density. The object of interest is

$$
\mathscr{A}(f, g)(x)=\left(1-\operatorname{th}^{2} x\right) \int_{|\operatorname{th} x| \leqq|y| \leqq 1} \frac{d y}{|y|} \frac{1}{1-y^{2}} \frac{1}{1-\frac{\operatorname{th}^{2} x}{y^{2}}} f\left(\operatorname{th}^{-1}(y)\right) g\left(\operatorname{th}^{-1}\left(\frac{\operatorname{th} x}{y}\right)\right) .
$$

Note that $\mathscr{A}(f, g)=\mathscr{A}(g, f)$ and that $\tilde{f}=\mathscr{S}(f)=\mathscr{A}(f, f)$, and hence, $D \mathscr{S}_{f} g$ $=2 \mathscr{A}(f, g)$.

Lemma 6.3. Assume that the functions $f, g$ are even, that $f, g \in L_{1} \cap L_{\infty}$, and $\|f\|_{1}=1,\|f\|_{\infty} \geqq 2$. Then

$$
\|\mathscr{A}(f, g)\|_{1} \leqq\|g\|_{1},
$$

and

$$
\|\mathscr{A}(f, g)\|_{2} \leqq \mathcal{O}(1)\left(\|g\|_{1}\|f\|_{\infty}+\|g\|_{\infty} \log \|f\|_{\infty}\right) .
$$

This is part of Lemma A2.

We need some information about the variance of $\mathscr{S}(f)$ when $f=\mathscr{S}_{1}(s, \varphi)$, $(s, \varphi) \in \mathscr{B}$. If $(s, \varphi) \in \mathscr{B}$, we can form

$$
f(x)=\mathscr{S}_{1}(s, \varphi)(x)=\left(\frac{n}{s}\right)^{1 / 2}\left\{h\left(\left(\frac{n}{s}\right)^{1 / 2} x\right)+\varphi\left(n^{1 / 2} x\right)\right\} .
$$

The variance of $\mathscr{S}(f)$ is then

$$
\tilde{\sigma}^{2}=\frac{n}{s} \int d x d y T(x, y)^{2}\left\{h\left(\left(\frac{n}{s}\right)^{1 / 2} x\right)+\varphi\left(n^{1 / 2} x\right)\right\}\left\{h\left(\left(\frac{n}{s}\right)^{1 / 2} y\right)+\varphi\left(n^{1 / 2} y\right)\right\},
$$

with $T(x, y)=\mid \operatorname{th}^{-1}(\operatorname{th}(x)$ th $(y)) \mid$. We change variables and get

$$
\tilde{\sigma}^{2}=\frac{1}{s} \int d x d y T\left(\frac{x}{n^{1 / 2}}, \frac{y}{n^{1 / 2}}\right)^{2}\left(h\left(\frac{x}{s^{1 / 2}}\right)+\varphi(x)\right)\left(h\left(\frac{y}{s^{1 / 2}}\right)+\varphi(y)\right) .
$$

By Lemma A3, $\tilde{\sigma}^{2} \leqq 25\left(\int x^{2}|f(x)| d x\right)^{2}$. But

$$
\begin{aligned}
\int x^{2}|f(x)| d x & \leqq \frac{s}{n}+\int x^{2}\left(\frac{n}{s}\right)^{1 / 2}\left|\varphi\left(n^{1 / 2} x\right)\right| d x \\
& \leqq \frac{s}{n}+\frac{2}{n s^{1 / 2}}\left[\|\varphi\|_{\infty}+\left\|x^{4} \varphi\right\|_{2}\right]
\end{aligned}
$$

by Lemma A5. Hence, we have shown

Lemma 6.4. If $(s, \varphi) \in \mathscr{B}$, then the variance $\tilde{\sigma}$ of $\mathscr{S}_{1}(s, \varphi)$ is bounded as follows: $\tilde{\sigma}\left(\mathscr{S} \mathscr{S}_{1}(s, \varphi)\right) \leqq \mathcal{O}(1 / n)$.

We will also need a lower bound on $\tilde{\sigma}$, see Lemma A6. 
Lemma 6.5. There is a constant $K>0$ such that for every $(s, \varphi) \in \mathscr{B}$ one has $\tilde{\sigma}\left(\mathscr{S} \mathscr{S}_{1}(s, \varphi)\right)>K / n$.

Proposition 6.6. The operator $\mathscr{S}_{1}$ is defined and analytic on $\mathscr{B}$, and $\mathscr{S}$ is defined on $\mathscr{S}_{1} \mathscr{B}$. For every $(s, \varphi) \in \mathscr{B}$ the tangent map $\left(D \mathscr{S} \mathscr{S}_{1}\right)_{(s, \varphi)}$ is a bounded linear operator from $\mathscr{H}$ to $\mathscr{K}$, with norm uniformly bounded in $n$. Furthermore, the variance $\tilde{\sigma}$ of $\mathscr{S}_{1}(s, \varphi)$ satisfies $2 / n \geqq \tilde{\sigma} \geqq 1 /(2 n)$.

Proof. The last statement is an easy consequence of going through the proofs of the two preceding lemmas. From the definition of $\mathscr{S}_{1}, \mathscr{H}$, and $\mathscr{H}_{1}$ it is obvious that

$$
\left\|\mathscr{S}_{1}\right\|_{\mathscr{B} \rightarrow \mathscr{H}_{1}} \leqq \mathcal{O}(1) \text {. }
$$

Notice that

$$
\begin{aligned}
{\left[D \mathscr{S}_{1,(s, \varphi)}(t, \psi)\right](x)=} & t \cdot\left\{-\frac{\mathscr{S}_{1}(s, \varphi)(x)}{2 s}-\frac{h^{\prime}\left(\left(\frac{n}{s}\right)^{1 / 2} x\right)\left(\frac{n}{s}\right)^{1 / 2} x}{2 s}\right\} \\
& +\left(\frac{n}{s}\right)^{1 / 2} \psi\left(n^{1 / 2} x\right),
\end{aligned}
$$

and hence,

$$
\left\|D \mathscr{S}_{1,(s, \varphi)}\right\|_{\mathscr{H}_{\rightarrow} \mathscr{H}_{1}} \leqq \mathcal{O}(1)
$$

as is easily checked.

We next analyze $\mathscr{S}$. If $f \in \mathscr{H}_{1}$, then

$$
\|f\|_{\infty} \leqq n^{1 / 2}|f|_{\mathscr{H}_{1}} \text { and }\left\|x^{4} f\right\|_{2} \leqq n^{-7 / 4}|f|_{\mathscr{H}_{1}} \text {. }
$$

It is easy to see from the definition of $\mathscr{S}_{1}$ that for $(s, \varphi) \in \mathscr{B},\left\|\mathscr{S}_{1}(s, \varphi)\right\|_{\infty}$ $=\mathcal{O}\left(n^{1 / 2}\right) \geqq 2$. We conclude from Lemma 6.3 that

$$
\|\mathscr{S} f\|_{1} \leqq\|f\|_{1}^{2} \leqq \mathcal{O}(1) \text { for } f \in \mathscr{S}_{1} \mathscr{B} \quad \text { by Lemma A5 }
$$

and

$$
\|\mathscr{S} f\|_{2} \leqq \mathcal{O}(1)\left\{\|f\|_{1}\|f\|_{\infty}+\|f\|_{\infty} \log \|f\|_{\infty}\right\} .
$$

By Lemma A7, $\left\|x^{4} \mathscr{S} f\right\|_{1} \leqq \mathcal{O}\left(\left\|x^{4} f\right\|_{2}^{2}\right)$. Hence, for $(s, \varphi) \in \mathscr{B}, \mathscr{S} \mathscr{S}_{1}(s, \varphi)$ is defined and $\left|\mathscr{S}_{\mathscr{S}_{1}}(s, \varphi)\right|_{\mathscr{K}} \leqq \mathscr{O}(1)$. Note that $D \mathscr{S}_{f} g=2 \mathscr{A}(f, g)$. Therefore, for $f \in \mathscr{S}_{1} \mathscr{B}$ and $g \in \mathscr{H}_{1}$, we find from Lemma 6.3 and Lemma A5

$$
\begin{aligned}
&\left\|D \mathscr{S}_{f} g\right\|_{1} \leqq \mathcal{O}\left(\|g\|_{1}\right) \leqq \mathcal{O}\left(n^{-1 / 2}\|g\|_{\infty}+n^{7 / 4}\left\|x^{4} g\right\|_{2}\right) \leqq \mathcal{O}\left(|g|_{\mathscr{H}_{1}}\right) \\
&\left\|D \mathscr{S}_{f} g\right\|_{2} \leqq \mathcal{O}\left(\|f\|_{\infty}\|g\|_{1}+\left(\log \|f\|_{\infty}\right) \cdot\|g\|_{\infty}\right) \\
& \\
& \leqq \mathcal{O}\left(n^{1 / 2}\right)\|g\|_{1}+\mathcal{O}\left(\log n^{1 / 2}\right)|g|_{\mathscr{H}_{1}} n^{1 / 2} \\
& \leqq \mathcal{O}\left(n^{1 / 2} \log n^{1 / 2}\right)|g|_{\mathscr{H}_{1}} .
\end{aligned}
$$

Finally, by Lemma A7 (using polarization), we have

$$
\left\|x^{4} D \mathscr{S}_{f} g\right\|_{1} \leqq \mathcal{O}\left(\left\|x^{4} f\right\|_{2}\left\|x^{4} g\right\|_{2}\right) \leqq \mathcal{O}\left(n^{-7 / 2}|g|_{\mathscr{H}_{1}}\right) \text {. }
$$

If we consider the definition of ||$_{\mathscr{K}}$, we see that the above estimates imply the assertion. Proposition 6.6 is proved. 
The Operator $\mathscr{T}$. The operator $\mathscr{T}$ corresponds to taking the sum of $n$ independent copies of the random variable $\tilde{\xi}$ whose density is $\widetilde{f}$. Basically, our approach consists in controlling the error terms in the central limit theorem. There is, of course, a vast literature associated with this problem, and our methods below are heavily inspired by it. But we are forced to redo the estimates again, for two reasons. First, our "density" $\tilde{f}$ need not be positive, when $s, \varphi$ vary in $\mathscr{B}$, and second, as a function of $n$ our bounds on various moments of $\tilde{f}$ are not totally uniform. Also, we need $L_{2}$ estimates which are not very common in the literature.

In the sequel, we find it more convenient to study directly the operator $\mathscr{T}_{1} \mathscr{T}$. (The operator $\mathscr{T}$ is anyway only needed in the definition of $\mathscr{N}$.) Note that $\mathscr{T}_{1}$ is linear, and thus, it is easy to verify the asserted properties of $\mathscr{T}$ from those of $\mathscr{T}_{1} \mathscr{T}$. We leave these details to the reader.

Our bounds on $\mathscr{T}_{1} \mathscr{T}$ are strongly influenced by those of Petrov [P]. The idea is to give bounds on the Fourier transform of $\tilde{f} \in \mathscr{K}$. These bounds are given for a "central" region (Lemma 6.7 below, cf. [P, p. 140]) and for an "external" region, cf. [S] (Lemma 6.8, below).

We introduce some notation. Given $\tilde{f} \in \mathscr{S} \mathscr{S}_{1} \mathscr{B} \subset \mathscr{K}$, we define $v=\mathscr{F} \tilde{f}$ as its Fourier transform and $\hat{f}_{n}(t)=v\left(t /\left(\sigma_{0} n^{1 / 2}\right)\right)^{n}$, where $\sigma_{0} \equiv 1 / n$. We also define

$$
\begin{gathered}
s \sigma_{0}^{2}=\int x^{2} \tilde{f}(x) d x=v^{\prime \prime}(0), \\
\beta_{4}=\int x^{4}|\tilde{f}(x)| d x, \\
\varrho_{4}=\beta_{4} /\left(s \sigma_{0}^{2}\right)^{2} .
\end{gathered}
$$

Finally, in order to study $D \mathscr{T}_{1} \mathscr{T}$, we consider for fixed $\tilde{f} \in \mathscr{K}$ a $\tilde{g} \in \mathscr{K}$ with $\int \tilde{g}(x) d x=0$. We set

$$
\hat{g}_{n}(t)=v\left(\frac{t}{\sigma_{0} n^{1 / 2}}\right)^{n-1}(\mathscr{F} \tilde{g})\left(\frac{t}{\sigma_{0} n^{1 / 2}}\right) .
$$

Note that $s$, as defined above, is, for $\tilde{f} \in \mathscr{S} \mathscr{S}_{1} \mathscr{B}$, a number in $[1 / 2,2]$ and that, by Proposition 6.6,

$$
\varrho_{4} \leqq n^{-7 / 2}|\tilde{f}|_{\mathscr{K}} \mathcal{O}\left(n^{4}\right)=\mathcal{O}\left(n^{1 / 2}\right) .
$$

Lemma 6.7. For sufficiently large $n$ one has uniformly in $n$ and in $|t|<\left(n / \varrho_{4} s\right)^{1 / 2}$ the following inequalities

$$
\begin{aligned}
& \left|\partial_{t}^{j}\left(\hat{f}_{n}(t)-e^{-s t^{2} / 2}\right)\right| \leqq \mathcal{O}\left(\varrho_{4} / n\right)\left(1+t^{10}\right) e^{-s t^{2} / 20} . \\
& \left|\partial_{t}^{j}\left(\hat{g}_{n}(t)-e^{-s t^{2} / 2} \frac{t^{2}}{2 \sigma_{0}^{2} n}(\mathscr{F} g)^{\prime \prime}(0)\right)\right| \\
& \quad \leqq \mathcal{O}(1)\left(1+\varrho_{4} / n^{1 / 2}\right) n^{-3 / 2}\left(1+t^{14}\right)|\tilde{g}|_{\mathscr{K}} e^{-s t^{2} / 20},
\end{aligned}
$$

for $j=0,1,2,3,4$.

Proof. We first prove 1) for $j=4$ and leave the cases 1) $j=0,1,2,3$ to the reader. A little algebra shows that

$$
\begin{aligned}
\partial_{t}^{4} \hat{f}_{n}(t)= & v(\tau)^{n-4} \frac{1}{n \sigma_{0}^{4}}\left[(n-1)(n-2)(n-3) v^{\prime}(\tau)^{4}+6(n-1)(n-2) v(\tau) v^{\prime}(\tau)^{2} v^{\prime \prime}(\tau)\right. \\
& \left.+3(n-1) v(\tau)^{2} v^{\prime \prime}(\tau)^{2}+4(n-1) v(\tau)^{2} v^{\prime}(\tau) v^{\prime \prime \prime}(\tau)+v(\tau)^{3} v^{\prime \prime \prime \prime}(\tau)\right],
\end{aligned}
$$


where $\tau=t / \sigma_{0} n^{1 / 2}$. From

$$
e^{i x}=1+i x-\frac{x^{2}}{2}-\frac{i x^{3}}{6}+x^{4} r(x), \quad \text { where } \quad|r(x)| \leqq \mathcal{O}(1), \quad \forall x \in \mathbb{R},
$$

we have

$$
\begin{gathered}
v\left(\frac{t}{\sigma_{0} n^{1 / 2}}\right)=1-\frac{s t^{2}}{2 n}+\frac{t^{4}}{n^{2}} \mathcal{O}\left(\varrho_{4}\right), \\
\left(\sigma_{0} n^{1 / 2}\right)^{-1} v^{\prime}\left(\frac{t}{\sigma_{0} n^{1 / 2}}\right)=-\frac{s t}{n}+\frac{t^{3}}{n^{2}} \mathcal{O}\left(\varrho_{4}\right), \\
\left(\sigma_{0} n^{1 / 2}\right)^{-2} v^{\prime \prime}\left(\frac{t}{\sigma_{0} n^{1 / 2}}\right)=-\frac{s}{n}+\frac{t^{2}}{n^{2}} \mathcal{O}\left(\varrho_{4}\right), \\
\left(\sigma_{0} n^{1 / 2}\right)^{-3} v^{\prime \prime \prime}\left(\frac{t}{\sigma_{0} n^{1 / 2}}\right)=\frac{t}{n^{2}} \mathcal{O}\left(\varrho_{4}\right), \\
\left(\sigma_{0} n^{1 / 2}\right)^{-4} v^{\prime \prime \prime \prime}\left(\frac{t}{\sigma_{0} n^{1 / 2}}\right)=\frac{1}{n^{2}} \mathcal{O}\left(\varrho_{4}\right) .
\end{gathered}
$$

Using $\varrho_{4} \geqq 1$ and $t \varrho_{4}^{1 / 2} n^{-1 / 2} \leqq 2$, it is easy to deduce

$$
\partial_{t}^{4} \hat{f}_{n}(t)=v(\tau)^{n-4}\left[t^{4} s^{4}-6 t^{2} s^{2}+3+\frac{\left(1+t^{6}\right)}{n} \mathcal{O}\left(\varrho_{4}\right)\right] .
$$

Next, we want to pass to $\log v$. We have

$$
v(\tau)=1-\frac{s t^{2}}{2 n}+\frac{s^{2} t^{4}}{n^{2}} \varrho_{4} r(t)=1-\frac{s t^{2}}{2 n}(1+\alpha),
$$

with $|r(t)| \leqq 1 / 24$ and hence, $|\alpha| \leqq 1 / 12$. Since

$$
\frac{s t^{2}}{2 n} \leqq \frac{s t^{2}}{2 n} \varrho_{4} \leqq 1 / 2, \quad \text { we find } \quad 1 \geqq v(\tau) \geqq \frac{11}{24} .
$$

Using now $\log (1-x)=-x-\int_{0}^{x} \frac{t d t}{1-t}$, we get

$$
\log v(\tau)=\log \left(1-\frac{s t^{2}}{2 n}(1+\alpha)\right)=-\frac{s t^{2}}{2 n}+\frac{s^{2} t^{4}}{n^{2}} \varrho_{4} q(t),
$$

with $|q(t)| \leqq \frac{1}{24}+\left(\frac{13}{24}\right)^{2} \frac{1}{2} \frac{24}{11} \leqq \frac{2}{5}$.

This implies

$$
v(\tau)^{n-4}=\exp \left(-\frac{(n-4)}{2 n} s t^{2}+\frac{(n-4) s^{2} t^{4}}{n^{2}} \varrho_{4} q(t)\right) .
$$

We now use the bound $\left|e^{x}-1\right| \leqq|x| e^{|x|}$, so that

$$
\left|v(\tau)^{n-4} \exp \left(\frac{1}{2} s t^{2}\right)-1\right| \leqq\left|\frac{2 s t^{2}}{n}+\frac{(n-4) s^{2} t^{4}}{n^{2}} \varrho_{4} q(t)\right| \cdot \exp \left(t^{2} s\left(\frac{2}{n}+\frac{2}{5}\right)\right) .
$$


This implies

$$
\left|\left(\frac{d}{d t}\right)^{4} v\left(\frac{t}{\sigma_{0} n^{1 / 2}}\right)^{n}-\left(s^{4} t^{4}-6 s^{2} t^{2}+3\right) e^{-s t^{2} / 2}\right| \leqq \mathcal{O}\left(\varrho_{4} / n\right)\left(1+t^{10}\right) e^{-s t^{2} / 20} .
$$

The assertion 1) follows now from $\partial_{t}^{4} e^{-s t^{2} / 2}=\left(s^{4} t^{4}-6 s^{2} t^{2}+3\right) e^{-s t^{2} / 2}$.

We now prove 2 ). A calculation similar to the preceding one shows that for $|t| \leqq\left(n / \varrho_{4} s\right)^{1 / 2}$ one has

$$
\left|\partial_{t}^{j}\left(v\left(\frac{t}{\sigma_{0} n^{1 / 2}}\right)^{n-1}-e^{-s t^{2} / 2}\right)\right| \leqq \mathcal{O}(1) \varrho_{4} n^{-1}\left(1+t^{10}\right) e^{-s t^{2} / 20}
$$

for $j=0,1,2,3,4$. We now observe [using $\mathscr{F} \tilde{g}(0)=0$ ] that with $w=\mathscr{F} \tilde{g}$ and $\tau=t / \sigma_{0} n^{1 / 2}$, one has

$$
w(\tau)=-\frac{t^{2}}{2 \sigma_{0}^{2} n} w^{\prime \prime}(0)+\frac{t^{4}}{\sigma_{0}^{4} n^{2}} \mathcal{O}\left(\sup _{u}\left|w^{\prime \prime \prime \prime}(u)\right|\right)=-\frac{t^{2}}{2 \sigma_{0}^{2} n} w^{\prime \prime}(0)+\frac{t^{4}}{n^{3 / 2}} \mathcal{O}\left(|\tilde{g}|_{\mathscr{K}}\right) .
$$

Similarly, for the derivatives:

$$
\left(\frac{d}{d t}\right)^{j} w\left(\frac{t}{\sigma_{0} n^{1 / 2}}\right)=-\partial_{t}^{j} t^{2} \frac{1}{2 \sigma_{0}^{2} n} w^{\prime \prime}(0)+\left(\partial_{t}^{j} t^{4}\right) \mathcal{O}\left(|\tilde{g}|_{\mathscr{K}} / n^{3 / 2}\right)
$$

for $j=1,2,3,4$. The assertion follows now from the chain rule and the bound (cf. Lemma A5):

$$
\frac{1}{\sigma_{0}^{2} n}\left|w^{\prime \prime}(0)\right| \leqq n^{-3 / 4}|\tilde{g}|_{\mathscr{K}}
$$

In the following lemma, we assume that $\tilde{f}$ has a decomposition $\tilde{f}=\tilde{f}_{0}+\tilde{\omega}$, with $\tilde{f}_{0}$ positive and $\tilde{\omega}$ small. More precisely, we assume

$$
\tilde{f}_{0} \geqq 0, \quad \int \tilde{f}_{0}(x) d x=1, \quad \tilde{\sigma}_{0}^{2}:=\int x^{2} \tilde{f}(x) d x=\frac{s}{n^{2}},
$$

with $s \in[1 / 2,2],\left\|\tilde{f}_{0}\right\|_{2} \leqq \mathcal{O}\left(n^{1 / 2} \log n\right)$, and $\varrho_{4}:=\left\|x^{4} \tilde{f}\right\|_{1} / \tilde{\sigma}^{4} \leqq \mathcal{O}\left(n^{1 / 2}\right)$. Finally, we assume $\|\tilde{\omega}\|_{1} \leqq \mathcal{O}\left(n^{-3 / 4}\right)$.

Remark 6.8. Let $\tilde{f} \in \mathscr{S} \mathscr{S}_{1} \mathscr{B}$. Then $\tilde{f}$ has a decomposition as above, with $\tilde{f}_{0}=\mathscr{S} \mathscr{S}_{1}\left(s_{0}, 0\right),\left(s_{0}, 0\right) \in \mathscr{B}$.

Proof. If $\left(s_{0}, \varphi\right) \in \mathscr{B}$, then $\mathscr{S}_{1}\left(s_{0}, \varphi\right)$ is a Gaussian plus a remainder, whose integral is zero. Applying the operator $\mathscr{S}$ (which we view as the bilinear expression $\mathscr{A}$ of Lemma 6.3), we see that $\tilde{f}_{0}=\mathscr{S} \mathscr{S}_{1}\left(s_{0}, 0\right)$ is a function of integral 1 , satisfying all of the above assumptions (use Lemma 6.3, Proposition 6.6, Lemma A7, and the definition of ||$\left._{\mathscr{K}}\right)$. Also, Lemma 6.3 implies the bound on $\|\tilde{\omega}\|_{1}$.

Lemma 6.9. Under the above assumptions on $\tilde{f}_{0}$ and $\tilde{\omega}$, one has

$$
\left|\mathscr{F} f\left(\frac{t}{\sigma_{0} n^{1 / 2}}\right)\right| \leqq e^{-n^{-2 / 3}} \quad \text { for } \quad|t| \geqq\left(n / \varrho_{4} S\right)^{1 / 2} .
$$


Proof. We shall first give a bound on $(\mathscr{F} \tilde{f})^{2}=\mathscr{F}(\tilde{f} * \tilde{f})$. We decompose $\tilde{f} * \tilde{f}$ $=\tilde{f}_{0} * \tilde{f}_{0}+2 \tilde{f}_{0} * \tilde{\omega}+\tilde{\omega} * \tilde{\omega}$. The second and third term are, respectively, bounded as

$$
\left\|\mathscr{F}\left(\tilde{f_{0}} * \tilde{\omega}\right)\right\|_{\infty} \leqq\left\|\tilde{f_{0}} * \tilde{\omega}\right\|_{1} \leqq\left\|\tilde{f}_{0}\right\|_{1} \cdot\|\tilde{\omega}\|_{1},
$$

and

$$
\|\mathscr{F}(\tilde{\omega} * \tilde{\omega})\|_{\infty} \leqq\|\tilde{\omega}\|_{1}^{2} .
$$

Consider now $\tilde{f_{0}} * \tilde{f_{0}}$. This function is nonnegative and has integral 1. By assumption

$$
C:=\left\|\tilde{f}_{0} * \tilde{f}_{0}\right\|_{\infty} \leqq\left\|\tilde{f}_{0}\right\|_{2}^{2} \leqq \mathcal{O}\left(n(\log n)^{2}\right) .
$$

We apply to $A=\mathscr{F}\left(\tilde{f_{0}} * \tilde{f_{0}}\right)$ the corollary of Lemma 1 of Statulyavichus [S], which states that

$$
|A(t)| \leqq \exp \left\{-\frac{t^{2}}{96 C^{2}\left(2 \tilde{\sigma}_{0}|t|+\pi\right)^{2}}\right\},
$$

where $\tilde{\sigma}_{0}^{2}=\int d x x^{2}\left(\tilde{f}_{0} * \tilde{f}_{0}\right)(x)$. By Lemma A6 and its proof we find $\tilde{\sigma}_{0}^{2}=2 \int d x x^{2} \tilde{f}_{0}(x)$ $\leqq 3 \tilde{\sigma}^{2}$. Therefore,

$$
|A(t)| \leqq \exp \left\{-\frac{t^{2}}{\mathcal{O}\left(n^{2}(\log n)^{4}\right)(4 \tilde{\sigma}|t|+\pi)^{2}}\right\} .
$$

Assuming $|t|>\left(n / \varrho_{4} s\right)^{1 / 2}$, we obtain

$$
\left|A\left(t / \sigma_{0} n^{1 / 2}\right)\right| \leqq \exp -\left\{\frac{\varrho_{4}^{-1} \sigma_{0}^{-2} S^{-1}}{\mathcal{O}\left(n^{2}(\log n)^{4}\right)\left(4 \varrho_{4}^{-1 / 2}+\pi\right)^{2}}\right\} .
$$

Recall that $\varrho_{4} \geqq 1$ and note, for later use, that by the assumption on $s$, we have \{\}$\leqq \mathcal{O}(1)$.

We now show that for $t$ in the required domain,

$$
\left|A\left(\frac{t}{\sigma_{0} n^{1 / 2}}\right)\right|+2\left\|\mathscr{F}\left(\tilde{f}_{0} * \tilde{\omega}\right)\right\|_{\infty}+\|\mathscr{F}(\tilde{\omega} * \tilde{\omega})\|_{\infty} \leqq C^{\prime}<1 .
$$

We use the inequality, valid for $\alpha, \beta>0, e^{-\alpha}+\beta=e^{-\alpha}\left(1+e^{\alpha} \beta\right) \leqq e^{-\alpha+e^{\alpha} \beta}$. We take $\alpha=\{\}$ and $\beta \leqq \mathcal{O}\left(\|\tilde{\omega}\|_{1}\left(\left\|\tilde{f}_{0}\right\|_{1}+\|\tilde{\omega}\|_{1}\right)\right)$, we find $\mathcal{O}(1) \geqq\{\} \geqq \mathcal{O}\left(\frac{n^{1 / 2}}{\log ^{4} n}\right)$ and $\beta \leqq \mathcal{O}\left(n^{-3 / 4}\right)$ from which the assertion follows.

In our next proposition, we still make the assumptions on $f_{0}$, given above, and we formulate the bounds on $\mathscr{T}_{1} \mathscr{T}$ and $D \mathscr{T}_{1} \mathscr{T}$.

Proposition 6.10. Define $f(x)=n^{1 / 2} \sigma_{0} \tilde{f}^{* n}\left(n^{1 / 2} \sigma_{0} x\right)$, where $\sigma_{0}=1 / n$. Then, for $n$ sufficiently large, one has

1) $f(x)=\frac{1}{\left(2 \pi s^{-1}\right)^{1 / 2}} e^{-s x^{2} / 2}+\varphi(x)$, with $s=\tilde{\sigma}^{2} \sigma_{0}^{-2}$ and $|\varphi|_{\mathscr{H}} \leqq \mathcal{O}\left(n^{-1 / 2}\right)$.

2) If $\tilde{g} \in \mathscr{K}$ and $\int \tilde{g}(x) d x=0$, then $g(x)=n^{1 / 2} \sigma_{0}\left(\tilde{f}^{*(n-1)} * \tilde{g}\right)\left(n^{1 / 2} \sigma_{0} x\right)$ satisfies

$$
\left|g-\frac{1}{2 n \sigma_{0}^{2} s^{1 / 2}} \int x^{2} \tilde{g}(x) d x\left(\partial^{2} h\right)\left(s^{-1 / 2} \cdot\right)\right|_{\mathscr{H}^{\prime}} \leqq \mathcal{O}\left(n^{-3 / 2}\right)|\tilde{g}|_{\mathscr{K}},
$$

and $\int g(x) d x=0$. 
Proof. From Lemma 6.7, we have, for $|t| \leqq\left(n / \varrho_{4} s\right)^{1 / 2}$,

$$
\left|\mathscr{F} f(t)-e^{-s t^{2} / 2}\right|+\left|\partial_{t}^{4}\left[(\mathscr{F} f)(t)-e^{-s t^{2} / 2}\right]\right| \leqq \mathcal{O}\left(\varrho_{4} / n\right)\left(1+t^{10}\right) e^{-s t^{2} / 3} .
$$

For $|t| \geqq\left(n / \varrho_{4} s\right)^{1 / 2}$, we have

$$
\left|\partial_{t}^{j} e^{-s t^{2} / 2}\right| \leqq e^{-n \varrho_{4}^{-1} / 4} \mathcal{O}(1)\left(1+t^{4}\right) e^{-t^{2} / 4} \quad \text { if } j \leqq 4 .
$$

Using Lemma 6.9 and Eq. (6.3) we also have

$$
\begin{aligned}
|\mathscr{F} f(t)|+\left|\partial_{t}^{4} \mathscr{F} f(t)\right| \leqq & \exp \left(-(n-6) n^{-2 / 3}\right) \cdot \mathcal{O}(1)\left|\mathscr{F} f\left(\frac{t}{\sigma_{0} n^{1 / 2}}\right)\right|^{2} \\
& \cdot\left(1+\frac{n^{4}}{\sigma_{0}^{4} n^{2}}\right)|\widetilde{f}|_{\mathscr{K}} .
\end{aligned}
$$

This implies, since $\varrho_{4} \leqq \mathcal{O}\left(n^{1 / 2}\right)$,

$$
|\varphi|_{\mathscr{H}^{\prime}} \leqq \mathcal{O}(1)|\tilde{f}|_{\mathscr{K}} n^{-1 / 2}+\mathcal{O}(1) e^{-n^{1 / 3}} .
$$

From the choice of $s$, we have immediately $\mathscr{F} f(0)=(\mathscr{F} f)^{\prime \prime}(0)=0$ and hence, 1$)$ is proven.

We now prove 2). If $|t| \leqq\left(n / \varrho_{4} s\right)^{1 / 2}$, then we deduce from Lemma 6.7,

$$
\begin{aligned}
X(t) & =\left|\mathscr{F} g(t)-\frac{t^{2}}{2 n \sigma_{0}^{2}}(\mathscr{F} g)^{\prime \prime}(0) e^{-s t^{2} / 2}\right|+\left|\left(\frac{d}{d t}\right)^{4}\left(\mathscr{F} g(t)-\frac{t^{2}}{2 n \sigma_{0}^{2}}(\mathscr{F} g)^{\prime \prime}(0) e^{-s t^{2} / 2}\right)\right| \\
& \leqq \mathcal{O}\left(n^{-3 / 2}\right)\left(1+t^{14}\right)|\tilde{g}|_{\mathscr{K}} e^{-s t^{2} / 3} .
\end{aligned}
$$

If $|t| \geqq\left(n / \varrho_{4} s\right)^{1 / 2}$, then by Lemma 6.9 we conclude that

This implies the assertion.

$$
X(t) \leqq \mathcal{O}\left(e^{-n^{1 / 3}}\right)\left|\tilde{f}\left(\frac{t}{\sigma_{0} n^{1 / 2}}\right)\right|^{2}|\tilde{g}|_{\mathscr{K}}
$$

Lemma 6.11. The matrix element $A$ of the "matrix" $D \mathscr{M}$ is equal to $2+\mathcal{O}\left(\frac{1}{n^{1 / 2}}\right)$.

Proof. A straightforward calculation shows that the matrix element $A$ equals

$$
A=n^{2} \int x^{2}\left[D \mathscr{S}_{1,(s, \varphi)}(1,0)\right](x) d x=2 n^{2} \int T(x, y)^{2} f(x) g(y) d x d y,
$$

with

$$
\begin{gathered}
f(x)=\left(\frac{n}{s}\right)^{1 / 2}\left[h\left(x\left(\frac{n}{s}\right)^{1 / 2}\right)+\varphi\left(x n^{1 / 2}\right)\right], \\
g(x)=n^{1 / 2} h\left(x n^{1 / 2}\right) .
\end{gathered}
$$

Note also that $s=1+\mathcal{O}\left(\frac{\log n}{n}\right)$. We consider first the case $s=1, \varphi=0$. Then

$$
\begin{aligned}
A & =2 n^{3} \int T(x, y)^{2} h\left(x n^{1 / 2}\right) h\left(y n^{1 / 2}\right) d x d y \\
& =2 n^{2} \int T\left(\frac{x}{n^{1 / 2}}, \frac{y}{n^{1 / 2}}\right)^{2} h(x) h(y) d x d y .
\end{aligned}
$$


We split the above integral as $\int_{|x|,|y| \leqq n^{1 / 4}}$ and its complement. The first term is expanded, using the Taylor expansion of $T^{2}$ as

$$
2 \int_{|x|,|y|<n^{1 / 4}} x^{2} y^{2} h(x) h(y) d x d y=2+\mathcal{O}\left(n^{-5}\right) .
$$

The remainder is $\mathcal{O}\left(n^{-5}\right)$, and the terms coming from $\varphi$ or from changing $s$ are at once bounded by Lemma A2.

Lemma 6.12. Given $\tilde{g} \in \mathscr{K}$, the first component of $D \mathscr{T}_{1} \mathscr{T}_{\tilde{f}} \tilde{g}$ is bounded by $\mathcal{O}\left(n^{1 / 4}\right)|\tilde{g}|_{k}$ uniformly in $\tilde{f} \in \mathscr{S} \mathscr{S}_{1} \mathscr{B}$.

Proof. We have, by definition

$$
\left(D \mathscr{T}_{1} \mathscr{T}_{\tilde{f}} \tilde{g}\right)_{\text {first comp. }}=n^{2} \int x^{2} \tilde{g}(x) d x .
$$

By the Schwarz inequality, this is bounded by

$$
n^{2}\left\|x^{4} \tilde{g}\right\|_{1}^{1 / 2}\|\tilde{g}\|_{1}^{1 / 2} \leqq \mathcal{O}\left(n^{2} n^{-7 / 4}\right)|\tilde{g}|_{\mathscr{K}},
$$

from which the assertion follows.

Proof of Theorem 6.2.1. This is really a variant of earlier estimates. Consider $(s, 0)$. The function $f=\mathscr{S}_{1}(s, 0)$ is Gaussian, and hence, by a variant of Lemma A6,

$$
\left\|x^{2 k} \tilde{f}\right\|_{1} \leqq C_{k} n^{-2 k} \quad \text { for } \quad k=0,1,2, \ldots, \text { i.e. }
$$

e.g. $\varrho_{4} \leqq \mathcal{O}(1)$ and not only $\mathcal{O}\left(n^{1 / 2}\right)$ as in the case of a general function in $\mathscr{B}$. Hence, by combining [P, Lemma 4, p. 140 of Petrov, for $s=10]$, and Lemma 6.9, we see that $\hat{f}(x)$ is a Gaussian $\left[\right.$ of variance $\left.1+\mathcal{O}\left(\frac{1}{n}\right)\right]$ plus a function $\hat{\varphi}$ which is $\mathcal{O}\left(\frac{1}{n}\right)$ in $\mathscr{H}^{\prime}$ [and not only $\mathcal{O}\left(n^{-1 / 2}\right)$ as in Proposition 6.10].

\section{Spin Observables}

In this section, we investigate the behavior of the expectation of the spin when the "volume" of the lattice tends to infinity. We fix the values of the spin at the two extreme points of the lattice, thereby choosing the boundary conditions. If $s$ is a spin which is not one of the above two, we shall denote by $\langle s\rangle$ its expectation for fixed boundary condition and fixed values of the random couplings. We show that the expectation $E\left(\langle s\rangle^{2}\right)$ (i.e. average over couplings) of $\langle s\rangle^{2}$ satisfies

$$
E\left(\langle s\rangle^{2}\right)=\left\{\begin{aligned}
0 & \text { at high temperature } \\
\neq 0 & \text { at low temperature }
\end{aligned}\right.
$$

and

$$
E(\langle s\rangle)=0 \quad \text { at all temperatures . }
$$

Note that the above argument shows a transition for the Edwards-Anderson $[\mathrm{T}, \mathrm{EG}]$ parameter. We shall also see that the value of the Edwards-Anderson parameter is independent of the boundary conditions. 


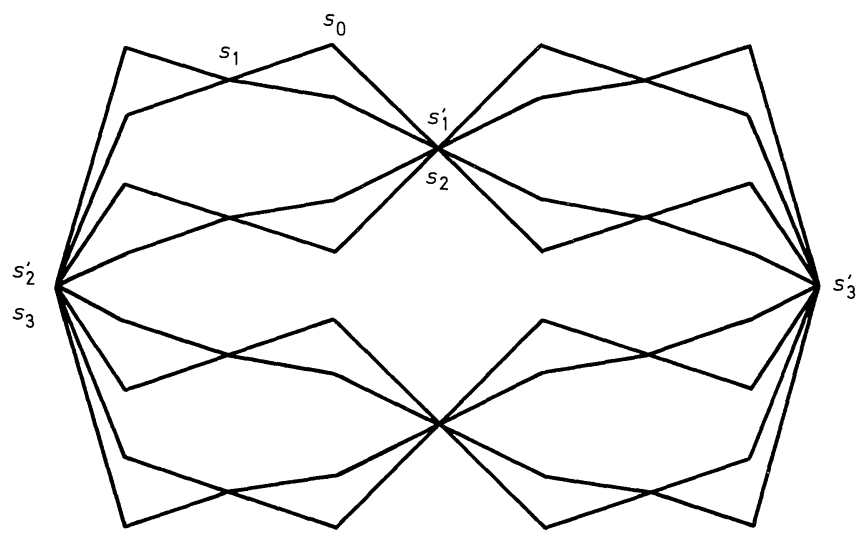

Fig. 5. Labelling of the spins

Consider the lattice $L_{N}$ and a fixed spin variable $s_{0}$ which has been "created" at level $N$. The variable $s_{0}$ has two neighboring sites, exactly one of which has been created at level $N-1$. We call it $s_{1}$ and we call the other neighbor $s_{1}^{\prime}$. Considering now $s_{1}$ as a fixed spin in $L_{N-1}$, we find its neighbors $s_{2}$ and $s_{2}^{\prime}$ in the same fashion as before, and continuing inductively, we find two chains $s_{0}, s_{1}, \ldots, s_{N}$ and $s_{0}, s_{1}^{\prime}, s_{2}^{\prime}, \ldots, s_{N}^{\prime}$ of spins. Note that all $s_{i}$ are distinct, but some of the $s_{i}^{\prime}$ may coincide. See Fig. 5.

Note also that either $s_{m+1}=s_{m}^{\prime}$ or $s_{m+1}^{\prime}=s_{m}^{\prime}$.

To these chains of spins, we associate functions of the form $F_{i}\left(s_{i}, s_{i}^{\prime}\right)=a_{i} s_{i}+b_{i} s_{i}^{\prime}$, where the $a_{i}, b_{i}$ are real functions depending on the couplings $x_{0}$. We are interested in $\left\langle F_{i}\left(s_{i}, s_{i}^{\prime}\right)\right\rangle$ [i.e. the canonical expectation of the observable $\left.F_{i}\left(s_{i}, s_{i}^{\prime}\right)\right]$. We have the following important identity: $\left\langle A s_{m}+B s_{m}^{\prime}\right\rangle=\left\langle A^{\prime} s_{m+1}+B^{\prime} s_{m+1}^{\prime}\right\rangle$, where

$$
\begin{aligned}
& A^{\prime}=B+A \frac{x_{m}\left(1-x_{m}^{\prime 2}\right)}{1-x_{m}^{2} x_{m}^{\prime 2}} \quad \text { if } s_{m+1}=s_{m}^{\prime}, \\
& B^{\prime}=A \frac{x_{m}^{\prime}\left(1-x_{m}^{2}\right)}{1-x_{m}^{2} x_{m}^{\prime 2}}
\end{aligned}
$$

and

$$
\begin{aligned}
& A^{\prime}=A \frac{x_{m}\left(1-x_{m}^{\prime 2}\right)}{1-x_{m}^{2} x_{m}^{\prime 2}} \quad \text { if } \quad s_{m+1}^{\prime}=s_{m}^{\prime} . \\
& B^{\prime}=B+A \frac{x_{m}^{\prime}\left(1-x_{m}^{2}\right)}{1-x_{m}^{2} x_{m}^{\prime 2}}
\end{aligned}
$$

Here, $x_{m}$ and $x_{m}^{\prime}$ are two identical, independent random couplings obtained from $x_{0}$ through $m$-fold application of the RG transformation, $x_{m}=\operatorname{th}\left(\mathscr{N}^{m} \xi\right)$. The above identities are immediately obtained by induction when summing over all spins at level $N-m$.

Namely,

$$
\left(1+x_{m} s_{m} s_{m+1}\right)\left(1+x_{m}^{\prime} s_{m} s_{m}^{\prime}\right)=\left(1+x_{m} x_{m}^{\prime} s_{m+1} s_{m+1}^{\prime}\right)\left(1+\frac{x_{m} s_{m+1}+x_{m}^{\prime} s_{m+1}^{\prime}}{1+x_{m} x_{m}^{\prime} s_{m+1} s_{m+1}^{\prime}} s_{m}\right)
$$


and, summing over $s_{m}$ (and taking the mean), we get

$$
\sum_{s_{m}}\left(1+\frac{x_{m} s_{m+1}+x_{m}^{\prime} s_{m+1}^{\prime}}{1+x_{m} x_{m}^{\prime} s_{m+1} s_{m+1}^{\prime}} s_{m}\right) \cdot\left(A s_{m}+B s_{m}^{\prime}\right)=A\left(\frac{x_{m} s_{m+1}+x_{m}^{\prime} s_{m+1}^{\prime}}{1+x_{m} x_{m}^{\prime} s_{m+1} s_{m+1}^{\prime}}\right)+B s_{m}^{\prime} .
$$

The equations follow from $s_{m}^{\prime}=s_{m+1}$ (respectively $=s_{m+1}^{\prime}$ ).

Given $s_{0}$ and $A_{0} \neq 0$, we therefore find, when $B_{0}=0$,

$$
\left\langle A_{0} s_{0}\right\rangle=\left\langle A_{1} s_{1}+B_{1} s_{1}^{\prime}\right\rangle=\ldots=\left\langle A_{N} s_{N}+B_{N} s_{N}^{\prime}\right\rangle,
$$

where the $A_{i}, B_{i}$ are recursively obtained by the above relations and are random variables in all $x_{j}, x_{j}^{\prime}=\operatorname{th}\left(\xi_{j}\right), \operatorname{th}\left(\xi_{j}^{\prime}\right)$ created above level $i$. We are interested in $E\left(\left\langle s_{0}\right\rangle^{2}\right)$, and this is equal to $E\left(A_{N}^{2}+B_{N}^{2}\right)+2 E\left(A_{N} B_{N}\right) s_{N} s_{N}^{\prime}$. We shall show below that at large $\beta, E\left(A_{N}^{2}+B_{N}^{2}\right)>1 / 2$ for all $N$ and that $2 E\left(A_{N} B_{N}\right)=0$. We further will show $E\left(A_{N}\right)=E\left(B_{N}\right)=0$. This clearly implies

Theorem 7.1. For large $\beta$, we have $E(\langle s\rangle)=0, E\left(\langle s\rangle^{2}\right)>1 / 2$, and $E\left(\langle s\rangle^{2}\right) \rightarrow 1$ as $\beta \rightarrow \infty$, for any site inside the lattice and any lattice size.

Remark 7.2. It is easy to see that for small $\beta, E\left(\langle s\rangle^{2}\right) \rightarrow 0$ as the lattice size goes to infinity.

Remark 7.3. Morally, Theorem 7.1 should hold for any temperature $\beta>\beta_{\text {crit }}$. We have, however, not worked out the crossover behavior, connecting Theorems 6.1 and 7.1. We believe this could be done in the style of [CE].

Remark 7.4. We comment on the meaning of $A_{N}$ and $B_{N}$. By the Eq. (7.3), $\left\langle s_{0}\right\rangle$ $=A_{N} s_{N}+B_{N} s_{N}^{\prime}$, with $A_{0}=1$. Here, $s_{0}$ is a spin "inside" the lattice, and $s_{N}$ and $s_{N}^{\prime}$ are the two extremal "outside" points of the lattice. Given a sample point in the probability space of couplings, $A_{N}$ and $B_{N}$ become numbers which tell us how the thermodynamic expectation of $\left\langle s_{0}\right\rangle$ is correlated to that of the "boundary conditions" $s_{N}$ and $s_{N}^{\prime}$. Note that by the definition of the model, every coupling is with equal probability positive or negative. Thus the averages over the sample space of these couplings, i.e. $E\left(A_{N}\right)$ and $E\left(B_{N}\right)$, are zero. But the fluctuations stay large for large $\beta$, i.e. $E\left(A_{N}^{2}+B_{N}^{2}\right)>1 / 2$, and they are small at high temperature. So a typical spin at low temperature "sees" long chains of strongly correlated neighbors, while at high temperature it does not. In this sense, our model represents a spin glass.

As a preparation for the study of $E\left(A_{N}^{2}+B_{N}^{2}\right)$ we need a few preliminary estimates. We shall use the following notation. We fix an $m \geqq 0$ and denote $A=A_{m}$, $B=B_{m}, A^{\prime}=A_{m+1}, B^{\prime}=B_{m+1}, x=x_{m}, y=x_{m}^{\prime}, \xi=\operatorname{th}^{-1}(x), \sigma=\sigma_{m}$. Note that by Theorem 4.1.1), $\sigma \rightarrow \infty$ exponentially fast with $m$. For definiteness, we assume that $A^{\prime}, B^{\prime}$ have been obtained from $A, B$ through relation (7.1). The other case is handled in total analogy.

Lemma 7.5. For $N>0, E\left(A_{N}\right)=E\left(B_{N}\right)=E\left(A_{N} B_{N}\right)=0$.

Proof. This proof is inspired by [ARS] and [SYG], who show the use of gauge symmetry. Denote by $\left\langle s_{0}\right\rangle_{s_{N}, s_{N}^{\prime}}(\xi)$ the expectation of $s_{0}$ with boundary conditions $s_{N}, s_{N}^{\prime}$ and couplings $\xi$. Since for $N>0$ none of the couplings connects $s_{N}$ to $s_{N}^{\prime}$, we have

$$
\left\langle s_{0}\right\rangle_{+,+}(\xi)=\left\langle s_{0}\right\rangle_{+-}\left(T_{+-} \xi\right)=\left\langle-s_{0}\right\rangle_{++}(\hat{T} \xi),
$$


where $T_{+-}$changes the sign of those interactions "connected" to $s_{N}^{\prime}$ and $\hat{T}$ those connected to $s_{0}$. Since the $\xi$ 's have distributions, (7.4) implies

$$
E\left(\left\langle s_{0}\right\rangle_{s_{N}, s_{N}^{\prime}}\right)=0, \quad \forall s_{N}, s_{N}^{\prime},
$$

and hence, $E\left(A_{N}\right)=E\left(B_{N}\right)=0$. But (7.4) also implies $E\left(\left\langle s_{0}\right\rangle_{+,+}^{2}\right)=E\left(\left\langle s_{0}\right\rangle_{+,-}^{2}\right)$ from which $E\left(A_{N} B_{N}\right)=0$ follows.

Lemma 7.6. $\left|A^{\prime}\right|+\left|B^{\prime}\right| \leqq|A|+|B|$.

Proof. Note that $|x|,\left|x^{\prime}\right| \leqq 1$. Hence,

$$
\begin{aligned}
\left|A^{\prime}\right|+\left|B^{\prime}\right| & \leqq|B|+|A| \frac{|x|\left(1-y^{2}\right)+|y|\left(1-x^{2}\right)}{\left(1-x^{2} y^{2}\right)} \\
& \leqq|B|+|A| \frac{|x|+|y|}{1+|x y|} \leqq|B|+|A| .
\end{aligned}
$$

Lemma 7.7. If $A_{0}=1, B_{0}=0$, then $\left|A_{m}\right|+\left|B_{m}\right| \leqq 1$ for all $m$.

Proof. Obvious from Lemma 7.6.

Lemma 7.8. For sufficiently large $\sigma$, we have

$$
E\left(A^{\prime 2}+B^{\prime 2}\right) \geqq\left(1-\sigma^{-1 / 2}\right) E\left(A^{2}+B^{2}\right)-\sigma^{-1 / 2} .
$$

Proof. We have

$$
E\left(A^{\prime 2}+B^{\prime 2}\right)=E\left(B^{2}\right)+E\left(A^{2} \frac{x^{2}\left(1-y^{2}\right)^{2}+y^{2}\left(1-x^{2}\right)^{2}}{\left(1-x^{2} y^{2}\right)^{2}}\right)
$$

since the crossed terms drop by Lemma 7.5. We define a set $\Omega$ (depending on $m$ ) as the subset in our sample space for which

$$
|\xi| \leqq \log \sigma \quad \text { or } \quad\left|\xi^{\prime}\right| \leqq \log \sigma \quad \text { or } \quad\left|\xi+\xi^{\prime}\right| \leqq \log \sigma \quad \text { or } \quad\left|\xi-\xi^{\prime}\right| \leqq \log \sigma .
$$

We shall show

(i) $\operatorname{Pr}(\Omega):=E\left(\chi_{\Omega}\right) \leqq \sigma^{-1 / 2}$.

(ii) On the complement $\Omega^{c}$ of $\Omega$,

$$
T:=\frac{x^{2}\left(1-y^{2}\right)^{2}+y^{2}\left(1-x^{2}\right)^{2}}{\left(1-x^{2} y^{2}\right)^{2}} \geqq 1-\sigma^{-1 / 2} .
$$

This implies the assertion of the lemma as follows:

$$
\begin{aligned}
E\left(A^{\prime 2}+B^{\prime 2}\right) & =E\left(B^{2}\right)+E\left(A^{2} T \chi_{\Omega^{c}}\right)+E\left(A^{2} T \chi_{\Omega}\right) \\
& \geqq E\left(B^{2}+A^{2} T \chi_{\Omega^{c}}\right) \\
& \geqq E\left(B^{2}+\left(1-\sigma^{-1 / 2}\right) A^{2} \chi_{\Omega^{c}}\right) \\
& \geqq E\left(B^{2}+\left(1-\sigma^{-1 / 2}\right) A^{2}\right)-E\left(A^{2} \chi_{\Omega}\right) \\
& \geqq\left(1-\sigma^{-1 / 2}\right) E\left(A^{2}+B^{2}\right)-\sigma^{-1 / 2}
\end{aligned}
$$

by Lemma 7.7 and (i). 
We next prove (i). By Theorem 4.1.2),

$$
\begin{aligned}
\operatorname{Pr}(|\xi|<\log \sigma) & \leqq 2 \int_{0}^{\log \sigma} d x\left(\frac{1}{\left(2 \pi \sigma^{2}\right)^{1 / 2}} e^{-x^{2} / 2 \sigma^{2}}+\frac{K \sigma^{2}}{n^{1 / 2}(\sigma+|x|)^{3}}\right) \\
& \leqq 1 / 4 \sigma^{-1 / 2} .
\end{aligned}
$$

Similarly, with $f$ denoting the density of $\xi$, we have

$$
\begin{aligned}
\operatorname{Pr}\left(\left|\xi+\xi^{\prime}\right|<\log \sigma\right) & =\int_{|x+y| \leqq \log \sigma} f(x) f(y) d x d y \\
& =\int f(x) d x \int_{-x-\log \sigma}^{-x+\log \sigma} f(y) d y \leqq 4(\log \sigma)\|f\|_{\infty} \\
& \leqq \mathcal{O}\left(\frac{\log \sigma}{\sigma}\right), \text { again by Theorem 4.1. }
\end{aligned}
$$

The case of $\left|\xi-\xi^{\prime}\right|$ is analogous, and thus, (i) follows. To prove (ii), we first note that on $\Omega^{c}$ one has $|\xi| \geqq \log \sigma$, and since $x=\operatorname{th}(\xi)$, this implies

$$
1-x^{2} \leqq 1 / \sigma .
$$

Of course, we also have on $\Omega^{c}$,

$$
1-y^{2} \leqq 1 / \sigma .
$$

We further observe that on $\Omega^{c}$,

$$
\frac{1-x^{2}}{1-y^{2}} \leqq \frac{1}{\sigma} \quad \text { or } \quad \frac{1-x^{2}}{1-y^{2}} \geqq \sigma .
$$

This is seen as follows: $\left|\xi+\xi^{\prime}\right| \geqq \log \sigma$ and $\left|\xi-\xi^{\prime}\right| \geqq \log \sigma$ imply that ||$\xi|-| \xi^{\prime}|| \geqq \log \sigma$. This in turn implies

$$
\left|\log \frac{1-|x|}{1-|y|}\right|>\log 2 \sigma .
$$

Combining now (7.6) with (7.7) and (7.9) implies the inequality (7.8).

Recall now the definition (7.5) of $T$. Since $x^{2}, y^{2} \leqq 1$, we have

$$
\begin{aligned}
T & =\frac{\left(1-y^{2}\right)^{2}+\left(1-x^{2}\right)^{2}}{\left(1-x^{2} y^{2}\right)^{2}}-2 \frac{\left(1-x^{2}\right)\left(1-y^{2}\right)}{\left(1-x^{2} y^{2}\right)^{2}}\left(2-x^{2} y^{2}\right) \\
& \geqq \frac{\left(1-y^{2}\right)^{2}+\left(1-x^{2}\right)^{2}}{\left(1-x^{2} y^{2}\right)}-2\left(1-x^{2}+1-y^{2}\right) \\
& =: U-2 V .
\end{aligned}
$$

On $\Omega^{c}$, we have, by (7.6) and (7.7), $0 \leqq V \leqq 2 / \sigma$, and

$$
\begin{aligned}
U & =\frac{\left(1-x^{2}\right)^{2}+\left(1-y^{2}\right)^{2}}{\left(\left(1-x^{2}\right)+\left(1-y^{2}\right)-\left(1-x^{2}\right)\left(1-y^{2}\right)\right)^{2}}=\frac{1+\left(\frac{1-y^{2}}{1-x^{2}}\right)^{2}}{\left(1+\frac{1-y^{2}}{1-x^{2}}-\left(1-x^{2}\right) \frac{1-y^{2}}{1-x^{2}}\right)^{2}} \\
& =\frac{1+\left(\left(1-y^{2}\right) /\left(1-x^{2}\right)\right)^{2}}{\left(1+x^{2}\left(1-y^{2}\right) /\left(1-x^{2}\right)\right)^{2}} .
\end{aligned}
$$


Now (7.8) implies $\left(1-x^{2}\right) /\left(1-y^{2}\right) \leqq 1 / \sigma$ (or the opposite case, which is similar), and hence, $|U-1| \leqq \mathcal{O}\left(\sigma^{-1}\right)$. Thus, (ii) follows.

Proof of Theorem 7.1. The first assertion is obvious from Lemma 7.5. Define $\Sigma_{m}=E\left(A_{m}^{2}+B_{m}^{2}\right)$. By assumption $\Sigma_{0}=1$. Applying Lemma 7.8 repeatedly, we see that

$$
\Sigma_{m+1} \geqq\left(1-\sigma_{m}^{-1 / 2}\right) \Sigma_{m}-\sigma_{m}^{-1 / 2},
$$

and hence,

$$
\Sigma_{m} \geqq \prod_{j=0}^{m-1}\left(1-\sigma_{j}^{-1 / 2}\right)-\sum_{\ell=0}^{m-1} \sigma_{\ell}^{-1 / 2} .
$$

By Theorem 4.1.1), $\sigma_{j} \geqq \beta Q^{1 / 2}\left(n^{1 / 2} / 320\right)^{j}$ and hence, the assertion follows, and, in fact, $\Sigma_{m} \rightarrow 1$ as $\beta \rightarrow \infty$.

\section{Appendix}

In the following lemma, we summarize the estimates on the function $\operatorname{th}^{-1}(\operatorname{th}(x) \operatorname{th}(y))$ which will be needed throughout.

Lemma A1. Denote $T(x, y)=\left|\operatorname{th}^{-1}(\operatorname{th}(x) \operatorname{th}(y))\right|$. Then

1) $T(x, y) \leqq 5 \frac{|x|}{(1+|x|)^{1 / 2}} \frac{|y|}{(1+|y|)^{1 / 2}}, x, y \in \mathbb{R}$.

2) $T(x, y) \geqq \min (|x|,|y|)-\log 2$.

3) If $|x|,|y|<1 / 2$, then $T(x, y) \geqq 0.8|x||y|$.

4) $T(x, y) \leqq|x y|^{1 / 2}$.

Proof. 1), 4) Note that $|\operatorname{th}(x)| \leqq \min (|x|, 1)$, for all $x \in \mathbb{R}$, and that $\mid$ th $^{-1}(z)|\leqq 2| z \mid$, for all $|z|<0.95$. Thus, if $|x|,|y|<0.95$, we have $T(x, y) \leqq 2|x y|$. If $|x| \geqq 0.95$ and $|y|<0.95$, we use the general bound $T(x, y) \leqq \operatorname{th}^{-1}(|\operatorname{th}(y)|)=|y|$. Finally, if $|x|,|y| \geqq 0.95$, then we use the bound $T(x, y) \leqq\left|t^{-1}(\operatorname{th}(x))\right|,\left|\operatorname{th}^{-1}(\operatorname{th}(y))\right|$, i.e. $T(x, y) \leqq|x y|^{1 / 2}$. Combining these bounds implies 1) and 4).

2) We have $\mid$ th $x \mid \geqq 1-2 \exp (-2|x|)$, and hence,

$$
|\operatorname{th} x \cdot \operatorname{th} y| \geqq 1-2\left(e^{-2|x|}+e^{-2|y|}\right) \geqq 1-4 e^{-2 \min (|x|,|y|)} .
$$

Finally,

$$
\mid \text { th }^{-1}(t) \mid \geqq-\frac{1}{2} \log (1-|t|),
$$

and combining these bounds yield 2).

3) For $|x|<1 / 2,|\operatorname{th}(x)| \geqq 0.9|x|$. Hence,

$$
T(x, y) \geqq \operatorname{th}^{-1}(0.8|x y|) \geqq 0.8|x y| .
$$

Define

$$
\begin{aligned}
\mathscr{A}(f, g)(x)= & \left(1-\operatorname{th}^{2} x\right) \int_{|\operatorname{th} x| \leqq|y| \leqq 1} \frac{d y}{|y|} \frac{1}{1-y^{2}} \frac{1}{1-\frac{\operatorname{th}^{2} x}{y^{2}}} \\
& \cdot f\left(\operatorname{th}^{-1} y\right) g\left(\operatorname{th}^{-1}\left(\frac{\operatorname{th} x}{y}\right)\right) .
\end{aligned}
$$


Note that $\mathscr{A}(f, g)=\mathscr{A}(g, f)$.

Lemma A2. Assume $f, g$ are even, $f, g \in L_{1} \cap L_{\infty}$, and $\|f\|_{1}=1$. Then $\|\mathscr{A}(f, g)\|_{1}$ $\leqq\|g\|_{1}$ and

$$
\|\mathscr{A}(f, g)\|_{2} \leqq \mathcal{O}(1) \begin{cases}\|g\|_{1}\|f\|_{\infty}+\|g\|_{\infty} \log \|f\|_{\infty} & \text { if }\|f\|_{\infty} \geqq 2, \\ \left(\|g\|_{1}\|g\|_{\infty}\right)^{1 / 2}+\|g\|_{1}\|f\|_{\infty}^{1 / 2}+\|g\|_{\infty} & \text { if }\|f\|_{\infty} \leqq 2 .\end{cases}
$$

Proof. By the change of variables $u=\operatorname{th}^{-1} y, v=\operatorname{th}^{-1}\left(\frac{\operatorname{th} x}{y}\right)$, we see that

$$
\int|\mathscr{A}(f, g)|(x) d x \leqq \int|f(u)| d u \int|g(v)| d v
$$

which is the first inequality.

To prove the second inequality, we set $a=\operatorname{th} x$ and decompose

$$
\frac{1-a^{2}}{\left(1-y^{2}\right)\left(y^{2}-a^{2}\right)}=\frac{1+a}{(1+y)(y+a)}\left\{\frac{1}{1-y}+\frac{1}{y-a}\right\} \text {. }
$$

Set $S(z)=\left|f\left(\operatorname{th}^{-1}(z)\right)\right|, T(z)=\left|g\left(\operatorname{th}^{-1}(z)\right)\right|$. Then

$$
\begin{aligned}
|\mathscr{A}(f, g)|(x) & \leqq J=(1+a) 2 \int_{a}^{1} \frac{y d y}{(1+y)(y+a)} S(y) T(a / y)\left\{\frac{1}{1-y}+\frac{1}{y-a}\right\} \\
& =(1+a) 2\left(J_{1}+J_{2}\right) .
\end{aligned}
$$

By the change of variables $z=a / y$ in $J_{2}$, we obtain

$$
J_{2}=\int_{a}^{1} \frac{d y}{\left(1-y^{2}\right)(y+a)} T(y) S(a / y) .
$$

Thus, we are led to bound expressions of the form

$$
J_{0}(x)=\int_{a}^{1} \frac{d y}{\left(1-y^{2}\right) y} U(y) V(a / y),
$$

with either $U=S, V=T$ or $U=T, V=S$. We set $\varepsilon=\min \left(1 / 2,\|f\|_{\infty}^{-1}\right)$. Assume for the moment $U=S, V=T$. For $a>\varepsilon$, we have

$$
J_{0}(x) \leqq\|V\|_{\infty} \cdot \frac{1}{\varepsilon} \int_{a}^{\infty} \frac{d y}{1-y^{2}} U(y)=\frac{1}{\varepsilon}\|g\|_{\infty}\|f\|_{1} .
$$

Thus,

$$
\begin{aligned}
\int_{\operatorname{th}^{-1}(\varepsilon)}^{\infty} d x\left|J_{0}(x)\right|^{2} & \leqq \mathcal{O}(1) \int_{\operatorname{th}^{-1}(\varepsilon)}^{\infty}|\mathscr{A}(f, g)| \frac{1}{\varepsilon}\|g\|_{\infty}\|f\|_{1} d x \\
& \leqq \mathcal{O}(1) \frac{1}{\varepsilon}\|f\|_{1}^{2}\|g\|_{\infty}\|g\|_{1} .
\end{aligned}
$$

In summary, if $U=S, V=T$, we get a bound $\mathcal{O}\left(\frac{1}{\varepsilon}\right)\|f\|_{1}^{2}\|g\|_{\infty}\|g\|_{1}$, while if $U=T$, $V=S$, we get $\mathcal{O}\left(\frac{1}{\varepsilon}\right)\|g\|_{1}^{2}\|f\|_{\infty}\|f\|_{1}$. 
Next consider $a<\varepsilon$. We split the integral $\int^{1}$ as $\int^{\varepsilon}+\int^{1}$. The second integral is bounded as before, and we consider the contribution of the first to the $L_{2}$ norm.

Consider first the case $U=S, V=T$. We have to bound

$$
I=\int_{0}^{\mathrm{th}^{-1}(\varepsilon)} d x \int_{a}^{\varepsilon} \frac{d u}{u} S(u) T\left(\frac{a}{u}\right) \int_{a}^{\varepsilon} \frac{d u^{\prime}}{u^{\prime}} S\left(u^{\prime}\right) T\left(\frac{a}{u^{\prime}}\right) .
$$

This is bounded by

$$
\|f\|_{\infty}^{2}\|g\|_{\infty} \int_{0}^{\mathrm{th}^{-1}(\varepsilon)} d x \int_{a}^{\varepsilon} \frac{d u}{u} \int_{a}^{\varepsilon} \frac{d u^{\prime}}{u^{\prime}} T\left(\frac{a}{u^{\prime}}\right) .
$$

Setting $z=\frac{1}{\varepsilon}$ th $x, \tilde{u}=u / \varepsilon, \tilde{u}^{\prime}=\varepsilon z / u^{\prime}$, and integrating by parts, we get

$$
I \leqq \mathcal{O}(1)\|f\|_{\infty}^{2}\|g\|_{\infty} \varepsilon \int_{0}^{1} d z(1-\log z) T(z) .
$$

We split this latter integral into $\int_{0}^{\varepsilon}+\int_{\varepsilon}^{1}$, and we get

$$
I \leqq \mathcal{O}(1)\|f\|_{\infty}^{2}\|g\|_{\infty} \varepsilon\left[\|g\|_{1}+\varepsilon \log \varepsilon^{-1}\|g\|_{\infty}+\log \varepsilon^{-1}\|g\|_{1}\right] .
$$

Similarly, if $U=T, V=S$ we obtain a bound with $f, g$ interchanged. Having bounded the $L_{2}$ norms of $\int_{a}^{\varepsilon}$ and of $\int_{\varepsilon}^{1}$, the bound on the $L_{2}$ norm of $J_{0}$ follows readily from the Schwarz inequality.

Assume first that $\|f\|_{\infty} \geqq 2$. Then $\varepsilon=\|f\|_{\infty}^{-1}$, and we get

$$
\begin{aligned}
\|\mathscr{A}(f, g)\|_{2}^{2} \leqq & \mathcal{O}(1)\left\{\|g\|_{\infty}\|g\|_{1}\|f\|_{\infty}+\|g\|_{1}^{2}\|f\|_{\infty}^{2}+\|g\|_{\infty}^{2} \log \|f\|_{\infty}\right. \\
& \left.+\|f\|_{\infty}\|g\|_{\infty}\|g\|_{1} \log \|f\|_{\infty}+\|g\|_{\infty}^{2}\left[1+2 \log \|f\|_{\infty}\right]\right\} \\
\leqq & \mathcal{O}(1)\left[\|g\|_{1}^{2} \cdot\|f\|_{\infty}^{2}+\|g\|_{\infty}\|g\|_{1} \cdot\|f\|_{\infty} \log \|f\|_{\infty}+\|g\|_{\infty}^{2} \log \|f\|_{\infty}\right] \\
\leqq & \mathcal{O}(1)\left[\|g\|_{1}\|f\|_{\infty}+\|g\|_{\infty} \log \|f\|_{\infty}\right]^{2} .
\end{aligned}
$$

If $\|f\|_{\infty}<2$, then $\varepsilon=1 / 2$, and we get

$$
\begin{aligned}
\|\mathscr{A}(f, g)\|_{2}^{2} \leqq & \mathcal{O}(1)\left\{\|g\|_{\infty}\|g\|_{1}+\|g\|_{1}^{2}\|f\|_{\infty}+\|f\|_{\infty}^{2}\|g\|_{\infty}\|g\|_{1}\right. \\
& \left.+\|f\|_{\infty}^{2}\|g\|_{\infty}^{2}+\|g\|_{\infty}^{2}\|f\|_{\infty}\|f\|_{1}+\|g\|_{\infty}^{2}\|f\|_{\infty}^{2}\right\} \\
\leqq & \mathcal{O}(1)\left\{\|f\|_{\infty}\|g\|_{1}^{2}+\|g\|_{\infty}\|g\|_{1}+\|g\|_{\infty}^{2}\right\} .
\end{aligned}
$$

Lemma A3. One has the inequality

$$
\int T(x, y)^{2}|f(x) f(y)| d x d y \leqq 25\left(\int x^{2}|f(x)| d x\right)^{2},
$$

and, when $f(x) \geqq 0, \int f(x) d x=1$, then one has

$$
\int T(x, y)^{3} f(x) f(y) d x d y \leqq\left(\int x^{2} f(x) d x\right)^{3 / 2},
$$

where $T(x, y)=\left|\operatorname{th}^{-1}(\operatorname{th}(x) \operatorname{th}(y))\right|$. 
Proof. By Lemma A1.1) we find $T(x, y)^{2} \leqq 25 x^{2} y^{2}$, so that the first inequality follows. But Lemma A1.4) tells us $T(x, y)^{3} \leqq|x|^{3 / 2}|y|^{3 / 2}$, so that the left-hand side of the second inequality is bounded by $\left(\int|x|^{3 / 2} f(x) d x\right)^{2}$, which is in turn bounded by $\left(\int x^{2} f(x) d x\right)^{3}$ by the Hölder inequality.

Corollary A4. Set $\gamma=1 /(50 n)$. Then $E\left(\xi^{2}\right) \leqq \gamma$ implies $E\left((\mathscr{N} \xi)^{2}\right) \leqq E\left(\xi^{2}\right) / 2$.

Proof. Since $E\left((\mathscr{N} \xi)^{2}\right)^{1 / 2}=n^{1 / 2} E(\widetilde{\xi})^{1 / 2}$, the assertion is obvious from Lemma A3.

Lemma A5. For every $\alpha>0$, one has the inequalities

1) $\|\varphi\|_{1} \leqq 2 \alpha\|\varphi\|_{\infty}+2 \alpha^{-7 / 2}\left\|x^{4} \varphi\right\|_{2}$,

2) $\left\|x^{2} \varphi\right\|_{1} \leqq 2 \alpha^{3}\|\varphi\|_{\infty}+2 \alpha^{-3 / 2}\left\|x^{4} \varphi\right\|_{2}$.

Proof. 1) We have

$$
\int|\varphi(x)| d x \leqq 2 \alpha\|\varphi\|_{\infty}+\int_{|x|>\alpha} x^{4}|\varphi(x)| x^{-4} d x \leqq 2 \alpha\|\varphi\|_{\infty}+2\left\|x^{4} \varphi\right\|_{2} \alpha^{-7 / 2} .
$$

2) We have

$$
\int\left|x^{2} \varphi(x)\right| d x \leqq \alpha^{3}\|\varphi\|_{\infty}+\int_{|x|>\alpha} x^{4}|\varphi(x)| x^{-2} d x \leqq 2 \alpha^{3}\|\varphi\|_{\infty}+2 \alpha^{-3 / 2}\left\|x^{4} \varphi\right\|_{2} .
$$

Lemma A6. If $(s, \varphi) \in \mathscr{B}$, then $\int x^{2} \mathscr{S} \mathscr{S}_{1}((s, \varphi))(x) d x \geqq K / n^{2}$, for some universal constant $K>0$.

Proof. For $\varphi=0$, the left-hand side above is equal to

$$
\int d x d y T\left(\frac{x s^{1 / 2}}{n^{1 / 2}}, \frac{y s^{1 / 2}}{n^{1 / 2}}\right)^{2} h(x) h(y)=I .
$$

We bound $I$ from below by restricting the integration to $|x|,|y|<n^{1 / 5}$. For $n$ large we may apply Lemma A1.3 and get

$$
T\left(\frac{x s^{1 / 2}}{n^{1 / 2}}, \frac{y s^{1 / 2}}{n^{1 / 2}}\right)^{2} \geqq 0.64|x|^{2}|y|^{2} s^{2} / n^{2} .
$$

Thus,

$$
I \geqq \mathcal{O}\left(n^{-2}\right)\left(\int_{|x|<n^{1 / 5}} x^{2} h(x) d x\right)^{2}=\mathcal{O}\left(n^{-2}\right) .
$$

If $\varphi \neq 0$ we bound the variance in the case $s=1$ by

$$
I-\int d x d y T\left(\frac{x}{n^{1 / 2}}, \frac{y}{n^{1 / 2}}\right)^{2}[2 h(x)|\varphi(y)|+|\varphi(x) \varphi(y)|]=I-2 I_{1}-I_{2} .
$$

(The case $s \neq 1$ is left to the reader.) By Lemma A1.1),

$$
T\left(\frac{x}{n^{1 / 2}}, \frac{y}{n^{1 / 2}}\right)^{2} \leqq \frac{25}{n^{2}} \frac{x^{2} y^{2}}{\left(1+\frac{|x|}{n^{1 / 2}}\right)\left(1+\frac{|y|}{n^{1 / 2}}\right)} \leqq \frac{25}{n^{2}} x^{2} y^{2}
$$

Thus, the bounds on the integrals factorize. We have $\int x^{2} h(x) d x=\mathcal{O}(1)$ and, by Lemma A5.2),

$$
\int x^{2}|\varphi(x)| d x \leqq \mathcal{O}\left(\|\varphi\|_{\infty} n^{3 / 11}+\left\|x^{4} \varphi\right\|_{2} n^{-3 / 22}\right) \leqq \mathcal{O}\left(n^{-1 / 3}\right) .
$$


Hence, $2 I_{1}+I_{2} \leqq \mathcal{O}\left(n^{-2-1 / 3}\right)$, and the assertion follows.

Lemma A7. If, for some $k \geqq 1$, one has $\left\|x^{2 k} f\right\|_{2} \leqq \gamma$, then $\left\|x^{2 k} \tilde{f}\right\|_{1} \leqq \mathcal{O}\left(25^{k}\right) \gamma^{2}$.

Proof. We use Lemma A1.1). This yields a bound

$$
\begin{aligned}
\left\|x^{2 k} \tilde{f}\right\|_{1} & =\int T(x, y)^{2 k}|f(x) f(y)| d x d y \\
& \leqq 5^{2 k}\left[\int \frac{x^{2 k}}{(1+|x|)^{k}}|f(x)| d x\right]^{2} \\
& \leqq 5^{2 k}\left\|x^{2 k} f\right\|_{2}^{2} \cdot \int \frac{1}{(1+|x|)^{2 k}} d x,
\end{aligned}
$$

and the assertion follows.

Acknowledgements. We wish to thank H. Epstein and O. Lanford for helpful discussions. J.-P. E. wishes to thank the IHES for the warm hospitality, and P. C. acknowledges partial support from the Fonds National Suisse.

\section{References}

[ARS] Avron, J.E., Roepstorff, G., Schulman, L.S.: Ground state degeneracy and ferromagnetism in a spin glass. J. Stat. Phys. 26, 25 (1981)

[BB] Benyoussef, A., Boccara, N.: Real space renormalization group investigation of threedimensional Ising spin glasses. Phys. Lett. 93 A, 351-353 (1983); Existence of spin-glass phases for three and four dimensional Ising and Heisenberg model (to appear)

[CE] Collet, P., Eckmann, J.-P.: A renormalization group analysis of the hierarchical model in statistical mechanics. In: Lecture Notes in Physics, Vol. 74. Berlin, Heidelberg, New York: Springer 1978

[CEGM] Collet, P., Eckmann, J.-P., Glaser, V., Martin, A.: J. Stat. Phys. (submitted)

[CEL] Collet, P., Eckmann, J.-P., Lanford, O.E., III: Universal properties of maps on an interval. Commun. Math. Phys. 76, 211-254 (1980)

[ET] Van Enter, A.C., Griffiths, R.B.: The order parameter in a spin glass. Commun. Math. Phys. 90, 319 (1983)

[GK] Griffiths, R.B., Kaufman, M.: Spin systems on hierarchical lattices. Phys. Rev. B 26, 5022-5032 (1982)

[K] Kadanoff, L.P.: Notes on Migdal's recursion formulas. Ann. Phys. 100, 359-394 (1976)

[M] Migdal, A.D.: Phase transitions in gauge and spin lattice systems. Zh. Eksp. Teor. Fiz. 69, 1457-1465 (1975)

[P] Petrov, V.V.: Sums of independent random variables. Berlin, Heidelberg, New York: Springer 1975

[S] Statulyavichus, V.A.: Limit theorems for densities and asymptotic expansions for distributions of sums of independent random variables. Theor. Prob. Appl.10, 582-595 (1965)

[SK] Sherrington, D., Kirkpatrick, S.: Solvable model of a spin-glass. Phys. Rev. Lett. 35, $1792(1975)$

[SYG] Sütö, A., Yalcin, T., Gruber, C.: A probabilistic approach to the models of spin glasses. J. Stat. Phys. 31, 639 (1983)

[T] Toulouse, G.: Frustrations et désordres: problèmes nouveaux en mécanique statistique. Histoire des verres de spin. Congrès de la Société Française de Physique, Clermont-Ferrand 1981 (Les Editions de Physique 1982)

Communicated by T. Spencer

Received November 3, 1983 